\title{
CAMA
}

Centre for Applied Macroeconomic Analysis

\section{Diversifier or More? Hedge and Safe Haven Properties of Green Bonds During COVID-19}

\section{CAMA Working Paper 20/2021 February 2021}

\section{Muhammad Arif}

Department of Business Administration, Shaheed Benazir Bhutto University

\section{Muhammad Abubakr Naeem}

School of Economics and Finance, Massey University

Business Administration Department, Faculty of Management Sciences, ILMA University

\section{Saqib Farid}

School of Business and Economics, University of Management and Technology

\section{Rabindra Nepal}

Faculty of Business and Law, School of Business, University of Wollongong

Centre for Applied Macroeconomic Analysis, ANU

\section{Tooraj Jamasb}

Copenhagen School of Energy Infrastructure, Department of Economics, Copenhagen

Business School 


\section{Abstract}

Against the backdrop of the Covid-19 pandemic, this study explores the hedging and safehaven potential of green bonds for conventional equity, fixed income, commodity, and forex investments. We use the cross-quantilogram approach that provides a better understanding of the dynamic relationship between assets under different market conditions. Our full sample results show that the green bond index could serve as a diversifier asset for medium- and long-term equity investors. Besides, it can also serve as a hedging and safe haven instrument for currency and commodity investments. Moreover, the sub-sample analysis of the pandemic crisis period shows a heightened short- and medium-term lead-lag association between the green bond index and conventional investment returns. However, the green bond index emerges as a significant hedging and safe-haven asset for the long-term investors of conventional financial assets. Our results offer insights for long-term investors whose portfolios comprise conventional assets such as equities, commodities, forex, and fixed income securities. Further, our findings reveal the potential role that the green bond investments could play in global financial recovery efforts without compromising the low-carbon transition targets.

\section{Keywords}

Green bonds, hedge, safe-haven, cross-quantilogram, COVID-19

\section{JEL Classification}

G10, G11, G19, Q01

Address for correspondence:

(E) cama.admin@anu.edu.au

\section{ISSN 2206-0332}

The Centre for Applied Macroeconomic Analysis in the Crawford School of Public Policy has been established to build strong links between professional macroeconomists. It provides a forum for quality macroeconomic research and discussion of policy issues between academia, government and the private sector.

The Crawford School of Public Policy is the Australian National University's public policy school, serving and influencing Australia, Asia and the Pacific through advanced policy research, graduate and executive education, and policy impact. 


\title{
Diversifier or More?
}

\section{Hedge and Safe Haven Properties of Green Bonds During COVID-19}

\author{
Muhammad Arif \\ Department of Business Administration, Shaheed Benazir Bhutto University, \\ Shaheed Benazirabad, Pakistan \\ Email: marif@sbbusba.edu.pk \\ Muhammad Abubakr Naeem \\ School of Economics and Finance, Massey University, New Zealand \\ Business Administration Department, Faculty of Management Sciences, \\ ILMA University, Karachi, Pakistan \\ Email: m.naeem@massey.ac.nz \\ Saqib Farid \\ School of Business and Economics, University of Management and Technology, Pakistan \\ Email: saqib.farid@umt.edu.pk \\ Rabindra Nepal \\ Faculty of Business and Law, School of Business \\ University of Wollongong, Australia \\ Center for Applied Macroeconomic Analysis \\ Australian National University, Australia \\ Email: rnepal@uow.edu.au \\ Tooraj Jamasb \\ Copenhagen School of Energy Infrastructure, Department of Economics, \\ Copenhagen Business School, Denmark \\ Email: tj.eco@cbs.dk
}

\section{January 2021}

\begin{abstract}
Against the backdrop of the Covid-19 pandemic, this study explores the hedging and safehaven potential of green bonds for conventional equity, fixed income, commodity, and forex investments. We use the cross-quantilogram approach that provides a better understanding of the dynamic relationship between assets under different market conditions. Our full sample results show that the green bond index could serve as a diversifier asset for medium- and long-term equity investors. Besides, it can also serve as a hedging and safe haven instrument for currency and commodity investments. Moreover, the sub-sample analysis of the pandemic crisis period shows a heightened short- and medium-term lead-lag association between the green bond index and conventional investment returns. However, the green bond index emerges as a significant hedging and safe-haven asset for the long-term investors of conventional financial assets. Our results offer insights for long-term investors whose portfolios comprise conventional assets such as equities, commodities, forex, and fixed income securities. Further, our findings reveal the potential role that the green bond investments could play in global financial recovery efforts without compromising the low-carbon transition targets.
\end{abstract}


Keywords: Green bonds, hedge, safe-haven, cross-quantilogram, COVID-19 JEL Classifications: G10, G11, G19, Q01 


\section{Introduction}

COVID-19 pandemic represents a global case of the fragility of the financial markets and vulnerability of global financial markets to natural disasters and exceptional risks. For instance, during the pandemic, the equity markets in the US hit the circuit breaker four times in two weeks and the markets in Europe and Asia touched the rock bottom. Crude oil prices plunged below 20 USD per barrel, a historical low in the new century and the US benchmark for Oil, crude oil futures for West Texas Intermediate (WTI), on 20 April 2020 closed at (-37.67) USD. The cited examples reveal the impact of the pandemic on financial markets. Overall, financial markets around the globe tumbled as panic selling across the markets drove uncertainty and contagion.

Unlike the previous pandemics, the much larger impact of COVID-19 on the global economy and financial markets has already been felt globally. World Bank forecast shows that global GDP will contract by $5.2 \%$ in 2020 due to the pandemic situation (World Bank, 2020). Most countries will likely experience recession, and advanced economies are expected to shrink by 7\%. Roubini (2019) argues that the financial crisis originated from the pandemic is expected to be deeper but short-lived compared to the Global Financial Crisis (GFC) of 2007-2008. On the other hand, the driving factors behind this financial slowdown are much more convoluted than before. Historically, the influence of such pandemics on financial markets has been modest and temporary (Selmi and Bouoiyour, 2020). For example, the same year, the SARS outbreak took place Chinese equity market grew by $20 \%$.

On the contrary, in the early stages of the COVID-19 outbreak, stock markets worldwide lost $30 \%$ of their market value within a few weeks, and the speed of sell-off exceeded that of GFC. While considering the significant losses in financial markets in the aftermath of the COVID19 pandemic, it is vital for scholars and practitioners to once again understand the interactions 
between different financial assets to safeguard against exceptional risks. More importantly, the need for safe-haven assets during exceptional times has resurfaced, and the previous evidence also reinforces that benefit of diversification across asset classes considerably decrease during times of high volatility in the markets (Campbell et al., 2002).

Lately, green bonds are considered a potential avenue for portfolio diversification and have been widely embraced by issuers and investors under the notion of green finance in the financial markets. In general, green bonds differ markedly from conventional bonds. Also, in view of the environmental concerns, governments around the globe aim to move from fossil fuels to renewable energy sources. This transition to a low-carbon economy requires large amount of financial resources and investments in climate-friendly, low-carbon, and energyefficient projects (Reboredo et al., 2020). Moreover, catching up with the transition to a low carbon economy entails disinvestment in conventional energy and mobilizing financial sources for green projects (OECD, 2016), which can support climate policy objectives and improve the profile or financial performance of firms by promoting green innovations (Brzeszczyński et al., 2019; Huynh et al., 2020). Thus, within the sustainability-oriented financial community, the green bonds are increasingly popular because they channel financial resources from conventional energies to environment friendly projects (Reboredo and Ugolini, 2020).

Green bonds hold similar characteristics as conventional fixed-income corporate instruments; however, their proceeds are only utilized for environment-friendly projects. The present-day "green bond boom" is reinforced by the publication of the Green Bond Principles (GBP) by the International Capital Markets Association in 2014, which has enhanced the reputation and transparency concerning green bonds (Reboredo, 2018). Stock markets worldwide have introduced specific green bond segments. Consequently, the size of the green bond market has increased from 11bn USD in 2013 to 257.7bn USD at the end of 2019 (Climate Bonds Initiative, 2019). Also, in the context of the 2015 Paris Agreement- in which countries around 
the world agreed to ensure the transition towards a climate-resilient economy, the green bonds market is anticipated to grow further. Likewise, a stream of literature has highlighted the advantages of green bond issuance for both issuers and subscribers. For instance, Nanayakkara and Colombage (2019) show that green bonds trade at the premium of 63 basis points. Tang and Zhang (2020) found that green bond issue improves stock liquidity, firm value, and institutional ownership. Lautsi (2019) show abnormal returns around the issuance of green bonds. Additionally, Flammer (2020) found a positive impact of green bond issuance on the environmental and financial performance of the issuer firms, which further leads to an increasing number of green investors and green innovations.

While green bonds are considered as well-established sustainable investment avenue by climate-conscious investors, traditional investors also realize the potential financial benefits of the green instrument. Many investors, including mutual funds, pension funds, individual investors, and insurance companies, are shifting focus towards green bonds considering the significant impact posed by climate-related risks for companies. Also, implying the fact that green bonds can serve as a diversifier for traditional instruments, the proposition of green bonds as an effective hedger and the safe-haven asset is important for portfolio managers and other market participants. The recent evidence presented by Nguyen et al. (2020) also shows that green bonds hold significant diversification benefits for both stocks and commodities.

Contextualizing the above discussion, this study examines the potential role of green bonds as a diversifier, hedge, and safe-haven asset for conventional assets (e.g., stocks, bonds, and commodities), especially during the COVID-19. Since the idea of the safe-haven asset is rooted in the notion of investor loss aversion (Tversky and Kahneman, 1991), which expresses that investors are more concerned about minimizing losses than realizing associated gains in the periods of the economic downturn (Hwang and Satchell, 2010). Thus, investors look for safehaven assets that are negatively correlated or uncorrelated with traditional assets during 
financial turmoil periods. Commonly, studies show various assets, including gold, long term treasury bonds, currencies, and cryptocurrencies, have been utilized as effective hedgers and safe-haven assets (see, e.g., Baur and Lucey, 2010; Ranaldo and Söderlind, 2010; Flavin et al., 2014; Urquhart and Zhang, 2019). Given the evidence, many mainstream studies have explored the potential role of different assets such as gold, foreign exchange, and cryptocurrencies as potential hedgers and safe-haven assets, during the turbulent times of the COVID-19 pandemic (Ji et al., 2020; Colon et al., 2020; Dutta et al., 2020, among others). Similarly, considering the inherent diversification capability of the green bond, we test whether green bond constitutes as an effective hedge and safe-haven asset during COVID-19. We search for further evidence on this growing debate. The findings of the study have implications not only for environmentally conscious investors but general investors can also use them for formulating their risk management strategies.

The study offers various contributions to the literature. First, focusing on the relationship between green bonds and other traditional assets, we are the first to provide evidence on the hedging and safe-haven potential of green bonds during the COVID-19 pandemic. The implications of such analysis are not only important from the perspective of portfolio and risk management, but they also provide a chance to evaluate the long-term contribution of green bonds to sustainable financial markets and climate-friendly community goals, particularly during the turbulent times of the pandemic. Accordingly, we model the dynamic patterns between the green bond market and other financial markets using the cross-quantilogram approach of Han et al. (2016) because the underlying markets can simultaneously undergo different conditions. The approach allows estimating the directional dependence between green bonds and other financial assets across different quantiles. As suggested by Liu et al. (2016), the technique is more effective than traditional methods (e.g., quantile regression with dummy variables) in measuring directional dependence between two assets, particularly when the 
bivariate normality assumption for joint distribution does not hold. Also, the predictive nature of the results assists investors to better understand the dynamic relationship between green bonds and other financial markets under different market conditions. Second, we also gauge the volatility dynamics of different financial markets by estimating the jump-activity. Many studies suggest that jumps can depict the crash risk of a financial time series, and they hold vital information for asset allocation and risk management (Clements and Liao, 2017; Oliva and Renò, 2018; Bouri et al., 2020). Thus, the findings unveil the volatility dynamics of different financial markets, especially the green bond market during the pandemic.

The findings of the study show that the green bond index serves as a diversifier asset for medium and long-term equity investors. Moreover, it has the potential to be a hedging and safe haven instrument for currency and commodity investments. Moreover, the sub-sample analysis of the pandemic crisis period shows a heightened short- and medium-term lead-lag association between the green bond index and conventional investment returns. However, the green bond index emerges as a significant hedging and safe haven asset for long-horizon investors. Further, the rolling sample analysis exhibits a dynamic lead-lag association in quantile returns that decreases overtime for the parallel lower return quantiles of green bond index and equity investment. Moreover, an increasing trend in the divergent return quantiles indicates the decoupling of green bond index returns from the convention asset returns. These findings offer several implications for investors and decision-makers to minimize investment risk and enhance the investment in low-carbon assets.

The remainder of the paper is organized as follows: Section 2 reviews the literature. Section 3 describes the methodology. Section 4 presents the data used in the study. Section 5 presents the results of the analysis. Section 6 is conclusions and policy implications. 


\section{Literature Review}

The present paper relates to the literature that discusses the emergence and performance of green finance in financial markets and its role in shaping up sustainable and environmentally friendly investments. Many studies have explored the effectiveness of green bonds for funding the cost of climate change (Flaherty et al., 2017; Banga, 2019; Semmler et al., 2019). Tang and Zhang (2020) assert that stock prices respond positively to the issuance of green bonds, and consequently, stock liquidity and institutional ownership also improve. Also, Flammer (2020) finds a positive impact of green bonds on the environmental and financial performance of the issuer firms and argues that it leads to green innovation in the long-term for issuers. In addition, previous studies reveal mixed results about the performance of environmental investments. Ortas and Moneva (2013) show higher returns of clean-technology indices than conventional indexes, but with higher risk.

On the contrary, Climent and Soriano (2011) and Reboredo et al. (2017) report lower or similar returns and low-risk protection of green mutual funds parallel to conventional funds. Several studies show that green bonds trade at a premium (Barclays, 2015; Zerbid, 2019; Nanayakkara and Colombage, 2019), while others showcase that green bonds tend to experience lower returns and higher volatility (Pham, 2016; Hachenberg and Schiereck, 2018; Baker et al., 2018; Kapraun and Scheins, 2019; Bachelet et al., 2019).

Another strand in the literature documents the influence of energy price movements, in particular, oil prices on the stock prices of renewable energy companies (Henriques and Sadorsky, 2012; Broadstock et al., 2012; Kumar et al., 2012; Managi and Okimoto, 2013; Inchauspe et al., 2015). Similarly, others have documented spillovers, causality, and tail dependence between oil prices and clean-energy stocks (Sadorsky, 2012; Wen et al., 2014; Reboredo, 2015; Reboredo et al., 2017). 
A more related strand of literature to our study investigates the relationship between the green bond market and other financial markets. These studies explore the inter-dependencies between green bonds and other financial assets and related implications concerning price spillovers and diversification benefits. For instance, Reboredo (2018) found that the green bond market closely co-moves with treasury and corporate bond markets, but still provides diversification chances for investors in the energy and stock market. While analyzing volatility dynamics between different bond markets, Pham (2016) discloses evidence of volatility clustering in the green bond market and spillover transmission from the conventional bond market. Broadstock and Cheng (2019) argue that the relationship between green bonds and the black bond market is subject to financial conditions such as news-based sentiments concerning green bonds, volatility, energy prices, economic activity, and economic policy uncertainty.

Similarly, Febi et al. (2018) show significant and time decreasing influence of liquidity on the yield spread of green bonds. Reboredo and Ugolini (2020) investigate the price connectedness between the green bond market and other financial markets. Their findings indicate that green bonds are closely associated with currency and fixed-income markets, where the green bond market is a net receiver of volatility shocks from both the underlying markets. On the contrary, the bond market has weak linkages with stock, energy, and high yield corporate bond markets. In the same way, Reboredo et al. (2020) found strong connectedness between the green bond market and treasury and corporate bonds in the US and EU countries. Once again, the evidence supported that green bonds receive sizeable volatility spillovers from other bond markets.

Furthermore, Nguyen et al. (2020) study the time-varying association between green bonds and other financial markets. The findings suggest strong co-movement between green bonds, commodities, and clean energy stocks, while the high diversification benefit of green bonds is revealed against commodities and stocks. Finally, few studies have examined the potential of green bonds as diversifiers and hedgers. For example, Saeed et al. (2020) investigate the 
potential of green stocks and bonds for hedging dirty assets and Huynh et al. (2020) examine the diversifier function of green bonds. Nevertheless, the literature is largely silent on the potential of green bonds for hedging and safe-haven purposes against other financial assets especially in the COVID-19 context. The present paper is one of the limited studies to investigate this.

\section{Methodology}

\subsection{Testing for jumps}

Using the semi-parametric approach of Laurent et al. (2016), we can detect the existence of jumps. The approach allows checking for additive jumps in AR-GJR-GARCH models. The AR (1)-GJR-GARCH $(1,1)$ for random returns $\left(\mathrm{R}_{\mathrm{t}}\right)$ is described as follows:

$R_{t}=\lambda_{t}+\xi_{t-1}+\epsilon_{t}$

$\epsilon_{t}=\varsigma_{t X_{t}}$, where $X_{t}$ i.i.d. $\mathrm{N}(0,1)$

$S_{t}^{2}=\varpi+\rho \epsilon_{t-1}^{2}+\vartheta_{1} C_{t-1} \epsilon_{t-1}^{2}+\beta \zeta_{t}^{2}$

The error term and white noise process are denoted by $\epsilon_{t}$ and $X_{t}$ respectively. The conditional variance of $\mathrm{R}_{\mathrm{t}}$ is represented by $\varsigma_{t}^{2}$, where $C_{t-1}=1$ only if $\epsilon_{t-i}<0$. Further adding an independent jump component $\left(b_{t} I_{t}\right)$ to $\left(R_{t}\right)$, it can be described as follows:

$* \mathrm{R}_{\mathrm{t}}=\mathrm{R}_{\mathrm{t}}+\mathrm{b}_{\mathrm{t}} \mathrm{I}_{\mathrm{t}}$

In equation (4), observed returns are represented by $* \mathrm{R}_{\mathrm{t}}$, and dichotomous variable taking is denoted by $I_{t}$, which has the value of 1 if there is a jump and otherwise 0 . Finally, the jump size is described by bt. 
Next, following the Muler et al. (2009) and Muler and Yohai (2008), we derive the estimates for $\lambda_{t}$ and $\mathrm{R}_{\mathrm{t}}$, which are robust to potential jumps $\left(\mathrm{b}_{\mathrm{t}} \mathrm{I}_{\mathrm{t}}\right.$ ) and denoted by $\sim \lambda_{t}$ and $\sim \varsigma_{\mathrm{t}}$. Now considering the standardized returns on a particular day $(\mathrm{t})$ as:

$\sim \mathrm{G}_{\mathrm{t}}=\frac{* \mathrm{Rt}-\sim \lambda_{t}}{\sim \varsigma \mathrm{t}}$

The null hypothesis $\mathrm{H}_{0}: \mathrm{b}_{\mathrm{t}} \mathrm{I}_{\mathrm{t}}=0$ is used to trace the existence of jumps against the research hypothesis $\mathrm{H}_{1}: \mathrm{b}_{\mathrm{t}} \mathrm{I}_{\mathrm{t}} \neq 0$. Here null hypothesis is rejected if $\max _{T}|\sim \mathrm{Gt}|>J_{T, \mu}$, where max is the maximum of $|\sim \mathrm{Gt}|$ for $\mathrm{t}=1,,,, \mathrm{~T}$ and critical value is represented by $J_{T, \mu}$. In the case of the rejection of the null hypothesis following dummy variable is generated:

$\sim \mathrm{I}_{\mathrm{t}}=\mathrm{I}(|\sim \mathrm{Gt}|>\mathrm{Q})$

In the above equation, the indicator function $\mathrm{I}($.$) with \sim \mathrm{I}_{\mathrm{t}}$ is regarded as 1 , if there is a jump on that particular day.

\subsection{Directional predictability through Cross-Quantilogram}

In order to examine the safe-haven function of green bonds against other financial markets, we apply the cross-quantilogram approach introduced by Han et al. (2016). Building on the work Linton and Whang (2007), they propose a bivariate measure of predictability across quantiles of the distribution of a stationary time series. The extended quantilogram estimates the lead/lag dependence between quantiles of two given financial time series and tests the directional predictability from one series to another. In this study, we utilize the unconditional version of the technique, employed by Jiang et al. (2016), Baumohl and Lyocsa (2017), and Liu et al. (2020) to estimate the spillovers between the assets.

Let us assume for an explanation; we represent the continuous returns of two series as $\mathrm{yi,t}, \mathrm{i}=$ 1,2 and $\mathrm{T}=1,2, \ldots \mathrm{t}$, where index I denotes returns of green bonds or a corresponding asset. 
Both series are strictly assumed to be stationary with unconditional function $F_{i}($.$) and$ unconditional density function $\mathrm{f}_{\mathrm{i}}($.$) . The corresponding unconditional quantile function is$ given as $\left(q_{i}\left(\tau_{i}\right)=\inf \left\{v: F_{i}(v) \geq \tau_{i}\right\}\right.$ for $\tau_{i} \in(0,1)$. Also, for arbitrary pair $\tau=\left(\tau_{1}, \tau_{2}\right)$, we measure the dependence between two events $\{y 1, t \leq q 1, t(\tau 1)\}$ and $\{y 2, t \leq q 2, t(\tau 2)\}$ with integer $\mathrm{k}= \pm 1, \pm 2$. The resulting quantilogram is shown in (7):

$$
\rho_{\tau}(k)=\frac{E\left[\psi_{\tau 1}\left(y_{1, t}-q_{1, t}\left(\tau_{1}\right)\right) \psi_{\tau 2}\left(y_{2, t}-q_{1, t-k}\left(\tau_{2}\right)\right)\right]}{\sqrt{E \psi_{\tau 1}^{2}\left[\left(y_{1, t}-q_{1, t}\left(\tau_{1}\right)\right) \sqrt{\psi_{\tau 2}^{2}\left(y_{2, t}-q_{1, t-k}\left(\tau_{2}\right)\right)}\right]}}
$$

The description of cross-quantilogram in equation (7) can be understood as cross-correlation of a quantile-hit process. In case when two series are identical, the above-proposed crossquantilogram corresponds to the quantilogram of Linton and Whang (2007). Further, provided the unconditional measure of $q *_{i}\left(\tau_{i}\right)$, the cross-quantilogram of the sample can be presented as in (8):

$$
\rho_{\tau}(k)=\frac{\sum_{t=k+1}^{T} \psi_{\tau 1}^{2}\left(y_{1, t}-q *_{1, t}\left(\tau_{1}\right)\right) \psi_{\tau 2}^{2}\left(y_{2, t}-q *_{1, t-k}\left(\tau_{2}\right)\right)}{\sqrt{\sum_{t=k+1}^{T} \psi_{\tau 1}^{2}\left(y_{1, t}-q *_{1, t}\left(\tau_{1}\right)\right) \sqrt{\sum_{t=k+1}^{T} \psi_{\tau 2}^{2}\left(y_{2, t}-q_{1, t-k}\left(\tau_{2}\right)\right)}}}
$$

Equation (8) is effective to measure the magnitude of directional dependence between two time series. By definition, the value of the equation is limited by construction to $\rho^{*} \tau(k) \in[-1,1]$. For instance, if we consider the $y 1, t$ be the continuous returns of green bonds and $y 2, t$ the continuous returns of any other specific asset, the value of $\rho^{*} \tau(1)=0$ means that if the returns on the other asset is above (below) at a given quantile $\mathrm{q} 2(\tau 2)$ at time $\mathrm{t}-1$, then it does assist in predicting the return on the green bonds is below (above) a given quantile q1( $\tau 1)$ at time $t$. In this case, lead/lag parameter $k$ controls the delay in predictability in terms of days from one series to another. 
Consequently, the resultant statistical test is obtained $H_{0}: \rho \tau(1)=\ldots=\rho \tau(p)=0, H_{1}: \exists k, \rho \tau$ $(k) \neq 0, k=1,2 \ldots p$, here we detect directional spillovers from one event $\{y 2, t-k \leq q 2, t-k(\tau 2)$ $: k=1,2 \ldots p\}$ to other event $\{y 1, t \leq q 1, t(\tau 1)\}$. Also, following Han et al. (2016) we define the Ljung-Box type of test statistic as follows:

$Q_{\tau}^{*}(\rho)=T(T+1) \sum_{K=1}^{P} \frac{\rho_{k}^{2}(k)}{T-k}$

According to Politis and Romano (1994), the critical values are obtained using a stationary bootstrap procedure, where pseudo samples is extracted from blocks of data with random block length. ${ }^{1}$ Further, in this study, we examine the dependence between all quantiles pairs given by $\{0.05,0.10, \ldots, 0.95\}$. Hence, for each pair of given time series with associated $p$ values, we estimate 361 dependence measures. In order to resolve the problem of multiple hypotheses, the study utilizes Bonferroni correction for adjusting the significance level, thus leading to a significance level of $0.00013(0.05 / 361=0.0001385)$. We illustrate our results in the form of heat maps.

\section{Data and Descriptive Analysis}

We use representative equity, bond, commodity, and currency indices and investments to explore the diversification and hedging potential of the SandP green bond index. Specifically, we choose three regional indices: Morgan Stanley Capital International (MSCI) World, MSCI Europe, MSCI Pacific, and four country-specific indices, namely, S\&P 500 composite, FTSE 100, DAX 30 performance, and Shanghai A-share equity indices. Besides, the S\&P GSCI commodity index, Crude oil Brent and gold represent commodity investments. Further, from the currency and fixed income markets, we pick US dollar and conventional Bond indices as representative conventional financial investments.

\footnotetext{
${ }^{1}$ See Politis and Romano (1994) and Patton et al. (2009) for details of expected block size.
} 


\subsection{Descriptive statistics}

Table 1 presents the results of the descriptive statistics that show equity indices such as $\mathrm{S} \& \mathrm{P}$ 500 (0.042), DAX 30 (0.028), and MSCI world (0.027) provide the highest mean returns. In contrast, commodity investments such as the S\&P GSCI commodity index (-0.035) and crude oil (-0.023) produce the lowest average returns. These commodity investments also exhibit the highest variability in the mean returns.

Table 1 presents the skewness and kurtosis estimates that reveals that all the data series except for the US dollar index are negatively skewed and possess heavy tails. Further, highly significant test statistic values of the Augmented Dicky-Fuller confirms the stationarity of data. Lastly, we check the non-linearity assumption using the Jarque-Bera test that confirms the nonlinearity of all the data variables, indicating that the quantile-based analysis methods are most appropriate.

[Table 1 about here]

\subsection{Return jumps}

Figure 1 displays the return jumps in the selected variables series that indicate a significant change in the return of an asset at a given time. BOND exhibits the highest jump activity (172 jumps), and the lowest jumps occurrence (16 jumps) is observed in the USDXY. Moreover, we observe lower jump activity (22 jumps) in green bond index return than other investments except for FTSE100, USDXY, DAXINDX, and MSEROP, which show 15, 16, 19, and 20 jumps, respectively. Nevertheless, the negative jumps activity is less prevalent (63.63\% of total jumps) in the green bond index compared to other selected assets except for BOND, USDXY, and GOLD as these assets show negative jumps activity of $36.6 \%, 50.0 \%$, and $60.7 \%$, respectively. This observation indicates that extreme negative returns are less likely in the green bond investments compared to most of the equity and commodity investments under investigation. 
[Figure 1 about here]

Further, one can see the co-occurrence of jumps in all the returns series during the year 2011 that coincides with the deepening of the European debt crisis. Similarly, the presence of cojumps in the 2015-2016 period exhibits the effects of the Chinese financial crisis, and these jumps are more pronounced for the equity markets than other financial markets, i.e., bond and commodity markets. Lastly, the pandemic period that starts from early 2020 shows the incidence of extreme negative jumps in all the returns series, signifying the significant impact of the pandemic crisis on selected financial assets.

\section{Cross Quantilogram Analysis}

The Jarque-Bera test and the occurrence of extreme jumps in our selected sample reveal the non-linear nature of variables under investigation, endorsing the use of quantile based methodology to explore the diversification and hedging properties of green bond investment against conventional equity, bond, forex, and commodity investments. To this end, we apply the cross-quantilogram approach to ascertain the lead-lag relationship between green bond and other investments and presents the results in heat maps setting where $\mathrm{x}$ - and $\mathrm{y}$-axis denote green bonds other investments returns, respectively. The graphical presentation of results in heat maps offers a complete and concise representation of the association between two investments at different quantiles. Besides, a convenient colour scheme classifies the strength and direction of association where red, green, and blue colour symbolize a positive, neutral, and negative association. Moreover, in the heat map environment, the green bond is categorized as a safe haven investment in two cases; (1) if the entire heat map shows the complete disconnection between the green bond and other assets, i.e., only green and/or blue colour is present in the heat map (2) if the bottom left corner of the heat map shows a negative association between the green bond and other assets, i.e., presence of blue colour in the bottom left corner of the 
heat map. Further, we classify green bonds as a diversifier when its middle return quantiles show a disconnection with other assets under examination.

Figure 2 presents the full sample cross-quantilogram results that show the lead-lag association between green bond investment and other investments under different time horizons, i.e., in the short-, medium-, and long-term. Specifically, column 1 presents the one-day lead-lag association between the green bond and other investments that reveal the asymmetric relationship in different quantiles. Notably, red colour is omnipresent in the lower-left quantiles of all the heat maps except for CHSASHR and USDXY, where we observe the widespread presence of green and blue colours. This observation indicates that except for CHSASHR and USDXY, green bond investments do not serve as a safe-haven asset for conventional investments in bearish market conditions under a short-term investment horizon. Additionally, in middle return quantiles ( 0.4 to 0.6 ), green bond shows a relatively less pronounced association with conventional investments that indicates the diversifier potential of green bond investments under a short investment horizon. Nevertheless, leading equity indices like SPCOMP, FTSE100, DAXINDEX, and MSWRLD still positively co-move with green bond investments, signifying that green bond offers very limited diversification avenues for shortterm equity investors.

[Figure 2 about here]

Further, the second and third column of figure 2 shows the medium- and long-horizon results that also exhibit a positive association in the lower-left quantiles between the green bond index and all the considered equity investments, signifying that green bond investments do not possess safe-haven or hedging potential for equity investments except for CHSASHR. However, excluding the SPCOMP index, green colour is omnipresent in the middle return quantiles for medium and long-horizon heatmaps, indicating that green bond could serve as a diversifier asset for both medium and long-term equity investors. Moreover, we observe that 
the green bond index maintains a neutral association with all commodity and currency investments in the lower-left and middle return quantiles, revealing the safe-haven and diversification potential of green investments for sample commodity and currency investments. Overall, the full sample analysis reveals that the green bonds could serve as a diversifier asset for medium and long-term equity investors. Moreover, it could be a potential hedging and safehaven asset for currency and commodity investments. These findings complement the existing literature that shows weak or negative connectedness of green bond investments with commodity securities (Nguyen et al., 2020; Reboredo, 2018; Reboredo and Ugolini, 2020). More importantly, our analysis reveals that the substitutive nature of the green bond makes it a prime instrument for the transition into climate-friendly investments.

\subsection{Cross quantilogram analysis - Pandemic crisis period}

Next, we estimate the cross-quantilogram results for the ongoing COVID-19 pandemic period. The pandemic period is the first global market downturns events since establishing the green bond market, hence providing the opportunity to ascertain the potential of the green bond to serve as an alternate investment during market-wide turbulence. Recent studies show that generally propagated a safe haven; cryptocurrencies failed to provide any substantial safehaven possibilities during the pandemic crisis period (Conlon and McGee, 2020; Dutta et al., 2020; Kristoufek, 2020). On the contrary, the traditional safe-haven asset, gold, offered safehaven prospects during this time (Ji et al., 2020; Kristoufek, 2020). Therefore, we attempt to explore how the market contagion will affect the diversifier and safe-haven potential of green bond investments.

[Figure 3 about here]

Column 1 in Figure 3 presents the short-term relationship between the green bond index and conventional investments that shows an enhanced lead-lag association in the lower-left returns quantiles, indicating that green bond failed to provide any safe-haven opportunities during the 
market contagion. Moreover, we also observe an increased lead-lag association between the green bond index and conventional investment in the middle return quantiles, suggesting that, during the pandemic, the green bond losses its diversification potential for short-term investors. Nevertheless, we observe the complete absence of red colour in the heat map that shows the association between the green bond and USDXY that signifies the availability of potential safehaven avenues for the short-term currency investors in the green bond investments.

Further, column 2 presents the medium-term cross-quantilogram heat maps that exhibit an analogues lower quantiles lead-lag association between the green bond index and conventional investments as of short-term results. However, we observe a decline in lead-lag association for middle return quantiles, indicating that conventional investors operating in medium-term investment horizons have limited diversification opportunities in green bond investments. Furthermore, the last column of Figure 3 shows that the green bond index return is completely disassociated from all the conventional investments. This finding indicates a significant change in the long-term lead-lag association between the green bond and conventional investments, especially the conventional equity investments, signifying that green bond investments offered substantial hedging and diversification avenues for the long-term investors in the recent pandemic crisis period.

Altogether, the pandemic crisis results reveal a heightened short and medium-term lead-lag association between the green bond index and conventional investment returns, indicating the ineffectiveness of green bond investments in providing shelter to short and medium-term investors. However, the green bond index emerges as a significant hedging and diversification instrument for long-term investors during the market-wide turbulence caused by the pandemic crisis. This is a significant finding given the severity of recent financial turbulence and the concerns raised around the energy transition goals (Kuzemko et al., 2020). 


\subsection{Rolling window cross-quantilogram analysis}

The cross-quantilogram heat maps present a static lead-lag association between the green bond index and conventional investments. However, existing studies report a time-varying dependence structure between green bond investments and conventional asset classes (see e.g., (Reboredo, 2018; Reboredo and Ugolini, 2020). Accordingly, we estimate a rolling window lead-lag association between the green bond index and conventional investments and present the results in graphical format. We estimate the time-varying lead-lag association by applying a recursive sampling approach that uses a 22-day rolling window. The estimation process starts from the first 22 days window and continues until the last day of the sample period. Figure 4 presents the rolling window cross-quantilogram results wherein the left, middle and right columns contain the lower (0.05), middle (0.5), and upper (0.95) quantiles of green bond index and red, blue and green lines show the lower, middle and upper quantiles of conventional investments under investigation. The horizontal and vertical axis represents the time and quantile hits of the green bond index, respectively.

Figure 4 confirms the time-varying lead-lag association between the green bond index and conventional investments, as all the graphs depict a dynamic pattern of association. Column 1 (Figure 4) shows the dynamic correlation between lower quantiles of the green bond index and conventional investment return quantiles. For example, the parallel lower quantiles of all equity investments and green bond index show a dynamic association that decreases over time. This observation indicates that green bond returns maintain a weak association with conventional equity investments during market-wide turbulence; hence, providing hedging avenues for equity investors. Additionally, a closer look at the pandemic period shows a noticeable decline in the lead-lag association, suggesting that green bond could be an alternate investment during a financial crunch period. The decline in the association between the green bond index and conventional investments, such as BOND, USDXY, and commodity investments, is less 
pronounced for the pandemic period. Moreover, we observe an increasing association between opposing quantiles of green bond index and conventional equity investments, which shows a decoupling effect between green bond returns and conventional investments.

[Figure 4 about here]

Furthermore, the middle and upper return quantiles of the green bonds index also show a declining association with the parallel return quantiles of conventional investments except for USDXY and SPGSCI. The decline in the lead-lag association is more pronounced for sample equity investments and OIL in the parallel middle returns quantiles, making green bond investments prime diversification choices for investors of these securities.

\section{Conclusions and policy Implications}

In the wake of the pandemic crisis, the financial markets worldwide are experiencing unprecedented downturns, forcing investors to look for alternate investments that provide diversification and hedging opportunities. Besides, countries worldwide are implementing stimulus packages to reboot economies that raise concerns regarding the transition to the lowcarbon economy. During these uncertain times, the appraisal of possible diversification and hedging potential of green financial assets becomes a topic of peculiar interest for investors of conventional assets and economic decision-makers. Such analysis promises essential insights that could help design climate-friendly economic policies and portfolio investments.

Against the backdrop of the pandemic crisis, this study explores the diversification and hedging potential of green bond investments for the conventional financial asset belonging to equity, fixed income, forex, and commodity markets. To this end, we employ the cross-quantilogram approach that provides the benefit of estimating the lead-lag association between two assets at different return quantiles. Besides, the predictive nature of the cross-quantilogram estimates 
can provide a better understanding of the dynamic relationship between green bonds and other financial investments under different market conditions.

Our full sample results reveal that the green bond index could serve as a diversifier asset for medium and long-term equity investors. Moreover, it can be a hedging and safe-haven instrument for currency and commodity investments. The sub-sample analysis of the pandemic crisis period shows a heightened short and medium-term lead-lag association between the green bond index and conventional investment returns. However, the green bond index emerges as a significant diversifier for long-term investors. Further, the rolling sample analysis exhibits a dynamic lead-lag association in quantile returns that decrease overtime for the parallel lower return quantiles of the green bond index and equity investment. Moreover, an increasing trend in the divergent return quantiles indicates the decoupling of green bond index returns from the convention asset returns.

Our findings offer several implications for investors and decision-makers to minimize investment risk and enhance the investment in low-carbon assets. Specifically, equity investors operating in medium and long-term horizons can use green bond investments to diversify their portfolios. Similarly, the commodity and currency investors may hedge downside risk by including green bond investments in their portfolios. Moreover, our results also show that green bond issuers and holders can not only achieve low-carbon investment objectives, but they can also avoid extreme financial market turbulence.

On the other hand, the resilience of green bonds during the pandemic suggest that it could serve as a sustainable instrument to reboot the global economy. Thus, governments worldwide can allocate resources from their financial stimulus packages to this alternative investment to help them reboot their economies without sacrificing the low-carbon transition targets set in different initiatives such as the Paris agreement and sustainable development goals. 


\section{References}

Bachelet, M. J., Becchetti, L., and Manfredonia, S. (2019). The green bonds premium puzzle: The role of issuer characteristics and third-party verification. Sustainability, 11(4), 1098.

Banga, J. (2019). The green bond market: a potential source of climate finance for developing countries. Journal of Sustainable Finance and Investment, 9(1), 17-32.

Barclays, 2015. The cost of being green. Credit research. https://www.environmentalfinance.

com/assets/files/US_Credit_Focus_The_Cost_of_Being_Green.pdf.

Baker, M., Bergstresser, D., Serafeim, G., and Wurgler, J. (2018). Financing the response to climate change: The pricing and ownership of US green bonds (No. w25194). National Bureau of Economic Research.

Baumöhl, E., and Lyócsa, S̆. (2017). Directional predictability from stock market sector indices to gold: A cross-quantilogram analysis. Finance Research Letters, 23, 152-164

Baur, D. G., and Lucey, B. M. (2010). Is gold a hedge or a safe haven? An analysis of stocks, bonds and gold. Financial Review, 45(2), 217-229.

Bouri, E., Roubaud, D., and Shahzad, S. J. H. (2020). Do Bitcoin and other cryptocurrencies jump together?. The Quarterly Review of Economics and Finance, 76, 396-409.

Broadstock, D. C., Cao, H., and Zhang, D. (2012). Oil shocks and their impact on energy related stocks in China. Energy Economics, 34(6), 1888-1895.

Brzeszczyński, J., Ghimire, B., Jamasb, T., and McIntosh, G. (2019). Socially Responsible Investment and Market Performance: The Case of Energy and Resource Companies, The Energy Journal, 4(5), 17-72.

Broadstock, D. C., and Cheng, L. T. (2019). Time-varying relation between black and green bond price benchmarks: Macroeconomic determinants for the first decade. Finance Research Letters, 29, 17-22.

Campbell, R., Koedijk, K., and Kofman, P. (2002). Increased correlation in bear markets. Financial Analysts Journal, 58(1), 87-94.

CBI, 2020. Green Bonds Global State of the Market 2019. Climate Bonds Initiative in Association with HSBC Climate Change Centre of Excellence.

Colon, F., Kim, C., Kim, H., and Kim, W. (2020). The Effect of Political and Economic Uncertainty on the Cryptocurrency Market. Finance Research Letters, 101621.

Clements, A., and Liao, Y. (2017). Forecasting the variance of stock index returns using jumps and cojumps. International Journal of Forecasting, 33(3), 729-742.

Climent, F., and Soriano, P. (2011). Green and good? The investment performance of US environmental mutual funds. Journal of Business Ethics, 103(2), 275-287.

Dutta, A., Das, D., Jana, R. K., and Vo, X. V. (2020). COVID-19 and oil market crash: Revisiting the safe haven property of gold and Bitcoin. Resources Policy, 69, 101816.

Flaherty, M., Gevorkyan, A., Radpour, S., and Semmler, W. (2017). Financing climate policies through climate bonds-A three stage model and empirics. Research in International Business and Finance, 42, 468-479.

Flammer, C. (2020). Green bonds: effectiveness and implications for public policy. Environmental and Energy Policy and the Economy, 1(1), 95-128. 
Flavin, T. J., Morley, C. E., and Panopoulou, E. (2014). Identifying safe haven assets for equity investors through an analysis of the stability of shock transmission. Journal of international financial markets, institutions and money, 33, 137-154.

Hachenberg, B., and Schiereck, D. (2018). Are green bonds priced differently from conventional bonds?. Journal of Asset Management, 19(6), 371-383.

Han, H., Linton, O., Oka, T., and Whang, Y. J. (2016). The cross-quantilogram: Measuring quantile dependence and testing directional predictability between time series. Journal of Econometrics, 193(1), 251-270.

Heine, D., Semmler, W., Mazzucato, M., Braga, J. P., Flaherty, M., Gevorkyan, A., and Radpour, S. (2019). Financing Low-Carbon Transitions through Carbon Pricing and Green Bonds. The World Bank. Retrieved from https://elibrary.worldbank.org/doi/abs/10.1596/1813-9450-8991.

Hwang, S., and Satchell, S. E. (2010). How loss averse are investors in financial markets?. Journal of Banking and Finance, 34(10), 2425-2438.

Huynh, T. L. D., Hille, E., and Nasir, M. A. (2020). Diversification in the age of the 4th industrial revolution: The role of artificial intelligence, green bonds and cryptocurrencies. Technological Forecasting and Social Change, 159, 120188.

Inchauspe, J., Ripple, R. D., and Trück, S. (2015). The dynamics of returns on renewable energy companies: A state-space approach. Energy Economics, 48, 325-335.

Ji, Q., Zhang, D., and Zhao, Y. (2020). Searching for safe-haven assets during the COVID-19 pandemic. International Review of Financial Analysis, 101526.

Jiang, H., Su, J. J., Todorova, N., and Roca, E. (2016). Spillovers and directional predictability with a cross-quantilogram analysis: The case of US and Chinese agricultural futures. Journal of Futures Markets, 36(12), 1231-1255.

Kapraun, J., and Scheins, C. (2019, March). (In)-Credibly Green: Which Bonds Trade at a Green Bond Premium?. In Proceedings of Paris December 2019 Finance Meeting EUROFIDAI-ESSEC.

Kristoufek, L. (2020). Grandpa, grandpa, tell me the one about Bitcoin being a safe haven: Evidence from the COVID-19 pandemics. arXiv preprint arXiv:2004.00047.

Kumar, S., Managi, S., and Matsuda, A. (2012). Stock prices of clean energy firms, oil and carbon markets: A vector autoregressive analysis. Energy Economics, 34(1), 215-226.

Kuzemko, C., Bradshaw, M., Bridge, G., Goldthau, A., Jewell, J., Overland, I., Westphal, K. (2020). Covid-19 and the politics of sustainable energy transitions. Energy research and social science, $68,101685$.

Lautsi, M. (2019). Green bonds and cumulative abnormal return implications for corporations around green bond announcements.

Laurent, S., Lecourt, C., and Palm, F. C. (2016). Testing for jumps in conditionally Gaussian ARMA-GARCH models, a robust approach. Computational Statistics and Data Analysis, 100, 383-400.

Linton, O., and Whang, Y. J. (2007). The quantilogram: With an application to evaluating directional predictability. Journal of Econometrics, 141(1), 250-282.

Liu, C. S., Chang, M. S., Wu, X., and Chui, C. M. (2016). Hedges or safe havens-revisit the role of gold and USD against stock: a multivariate extended skew-t copula approach. Quantitative Finance, 16(11), 1763-1789. 
Liu, C., Naeem, M. A., Rehman, M. U., Farid, S., and Shahzad, S. J. H. (2020). Oil as Hedge, Safe-Haven, and Diversifier for Conventional Currencies. Energies, 13(17), 4354.

Managi, S., and Okimoto, T. (2013). Does the price of oil interact with clean energy prices in the stock market?. Japan and the World Economy, 27, 1-9.

Muler, N., Pena, D., and Yohai, V. J. (2009). Robust estimation for ARMA models. The Annals of Statistics, 37(2), 816-840.

Muler, N., and Yohai, V. J. (2008). Robust estimates for GARCH models. Journal of Statistical Planning and Inference, 138(10), 2918-2940.

Nanayakkara, M., and Colombage, S. (2019). Do investors in Green Bond market pay a premium? Global evidence. Applied Economics, 51(40), 4425-4437.

Nguyen, T. T. H., Naeem, M. A., Balli, F., Balli, H. O., and Vo, X. V. (2020). Time-Frequency comovement among Green Bonds, Stocks, Commodities, Clean Energy, and Conventional Bonds. Finance Research Letters, 101739.

OECD (2016). Mobilising the Debt Capital Markets for a Low Carbon Transition. OECD Publishing, Paris.

Oliva, I., and Renò, R. (2018). Optimal portfolio allocation with volatility and co-jump risk that Markowitz would like. Journal of Economic Dynamics and Control, 94, 242-256.

Ortas, E., and Moneva, J. M. (2013). The Clean Techs equity indexes at stake: Risk and return dynamics analysis. Energy, 57, 259-269.

Patton, A., Politis, D. N., and White, H. (2009). Correction to "Automatic block-length selection for the dependent bootstrap" by D. Politis and H. White. Econometric Reviews, 28(4), 372-375.

Pham, L. (2016). Is it risky to go green? A volatility analysis of the green bond market. Journal of Sustainable Finance and Investment, 6(4), 263-291.

Politis, D. N., and Romano, J. P. (1994). The stationary bootstrap. Journal of the American Statistical association, 89(428), 1303-1313.

Ranaldo, A., and Söderlind, P. (2010). Safe haven currencies. Review of finance, 14(3), 385407.

Reboredo, J.C., 2015. Is there dependence and systemic risk between oil and renewable. energy stock prices? Energy Econ. 48, 32-45.

Reboredo, J. C., Quintela, M., and Otero, L. A. (2017). Do investors pay a premium for going green? Evidence from alternative energy mutual funds. Renewable and Sustainable Energy Reviews, 73, 512-520.

Reboredo, J. C. (2018). Green bond and financial markets: Co-movement, diversification and price spillover effects. Energy Economics, 74, 38-50.

Reboredo, J. C., Ugolini, A., and Aiube, F. A. L. (2020). Network connectedness of green bonds and asset classes. Energy Economics, 86, 104629.

Reboredo, J. C., and Ugolini, A. (2020). Price connectedness between green bond and financial markets. Economic Modelling, 88, 25-38.

Roubini, N. (2019). The Growing Risk of a 2020 Recession and Crisis. Project Syndicate.

Sadorsky, P. (2012). Correlations and volatility spillovers between oil prices and the stock prices of clean energy and technology companies. Energy economics, 34(1), 248-255.

Saeed, T., Bouri, E., and Vo, X. V. (2020). Hedging Strategies of Green Assets against Dirty Energy Assets. Energies, 13(12), 3141. 
Selmi, R., and Bouoiyour, J. (2020). Global Market's Diagnosis on Coronavirus: A Tug of War between Hope and Fear.

Tang, D. Y., and Zhang, Y. (2020). Do shareholders benefit from green bonds?. Journal of Corporate Finance, 61, 101427.

Tversky, A., and Kahneman, D. (1991). Loss aversion in riskless choice: A referencedependent model. The quarterly journal of economics, 106(4), 1039-1061.

Urquhart, A., and Zhang, H. (2019). Is Bitcoin a hedge or safe haven for currencies? An intraday analysis. International Review of Financial Analysis, 63, 49-57.

Wen, X., Guo, Y., Wei, Y., and Huang, D. (2014). How do the stock prices of new energy and fossil fuel companies correlate? Evidence from China. Energy Economics, 41, 63-75.

World. Bank. 2020. The Global Economic Outlook During the COVID-19 Pandemic: A Changed World. https://www.worldbank.org/en/news/feature/2020/06/08/the-globaleconomic-outlook-during-the-covid-19-pandemic-a-changed-world

Zerbib, O. D. (2019). The effect of pro-environmental preferences on bond prices: Evidence from green bonds. Journal of Banking and Finance, 98, 39-60. 


\begin{tabular}{|c|c|c|c|c|c|c|c|}
\hline \multicolumn{7}{|c|}{ Table 1: Descriptive statistics and unit root } & \multirow[b]{2}{*}{ JB } \\
\hline & ABB & $\begin{array}{c}\text { Mean } \\
(\%)\end{array}$ & $\begin{array}{l}\text { SD } \\
(\%)\end{array}$ & Skew & Kurt & ADF & \\
\hline S\&P GREEN BOND INDEX & SPGRBND & -0.002 & 0.399 & -0.363 & 9.124 & $-28.667 * * *$ & $4090.21 * * *$ \\
\hline S\&P 500 COMPOSITE INDEX & SPCOMP & 0.042 & 1.075 & -0.950 & 22.537 & $-29.278 * * *$ & $41436.44 * * *$ \\
\hline FTSE 100 & FTSE100 & 0.003 & 1.019 & -0.814 & 14.739 & $-29.860 * * *$ & $15105.59^{* * * *}$ \\
\hline DAX 30 PERFORMANCE & DAXINDX & 0.028 & 1.278 & -0.593 & 11.718 & $-28.657 * * *$ & $8324.11 * * *$ \\
\hline SHANGHAI SE A SHARE & CHSASHR & 0.009 & 1.323 & -0.980 & 10.104 & $-28.975 * * *$ & $5840.64 * * *$ \\
\hline MSCI WORLD & MSWRLD & 0.027 & 0.940 & -1.208 & 21.804 & $-28.213 * * *$ & $38654.48^{* * * *}$ \\
\hline MSCI EUROPE & MSEROP & 0.006 & 1.199 & -0.989 & 15.146 & $-29.571 * * *$ & $16286.74 * * *$ \\
\hline MSCI PACIFIC & MSPACF & 0.010 & 0.989 & -0.309 & 7.276 & $-28.026 * * *$ & $2007.07 * * *$ \\
\hline BOND INDEX & BOND & 0.005 & 0.396 & -0.437 & 71.846 & $-29.237 * * *$ & $509809.10 * * *$ \\
\hline S\&P GSCI Commodity & SPGSCI & -0.035 & 1.297 & -0.865 & 12.987 & $-28.532 * * *$ & $11047.53^{* * * *}$ \\
\hline US DOLLAR INDEX & USDXY & 0.007 & 0.437 & 0.077 & 4.792 & $-28.880 * * *$ & $347.68^{* * *}$ \\
\hline CRUDE OIL BRENT & OIL & -0.023 & 2.862 & -3.382 & 132.457 & $-30.851 * * *$ & $1807229.00 * * *$ \\
\hline GOLD & GOLD & 0.016 & 0.977 & -0.731 & 11.111 & $-29.112 * * *$ & $7304.72 * * *$ \\
\hline
\end{tabular}


A) SPGRBND

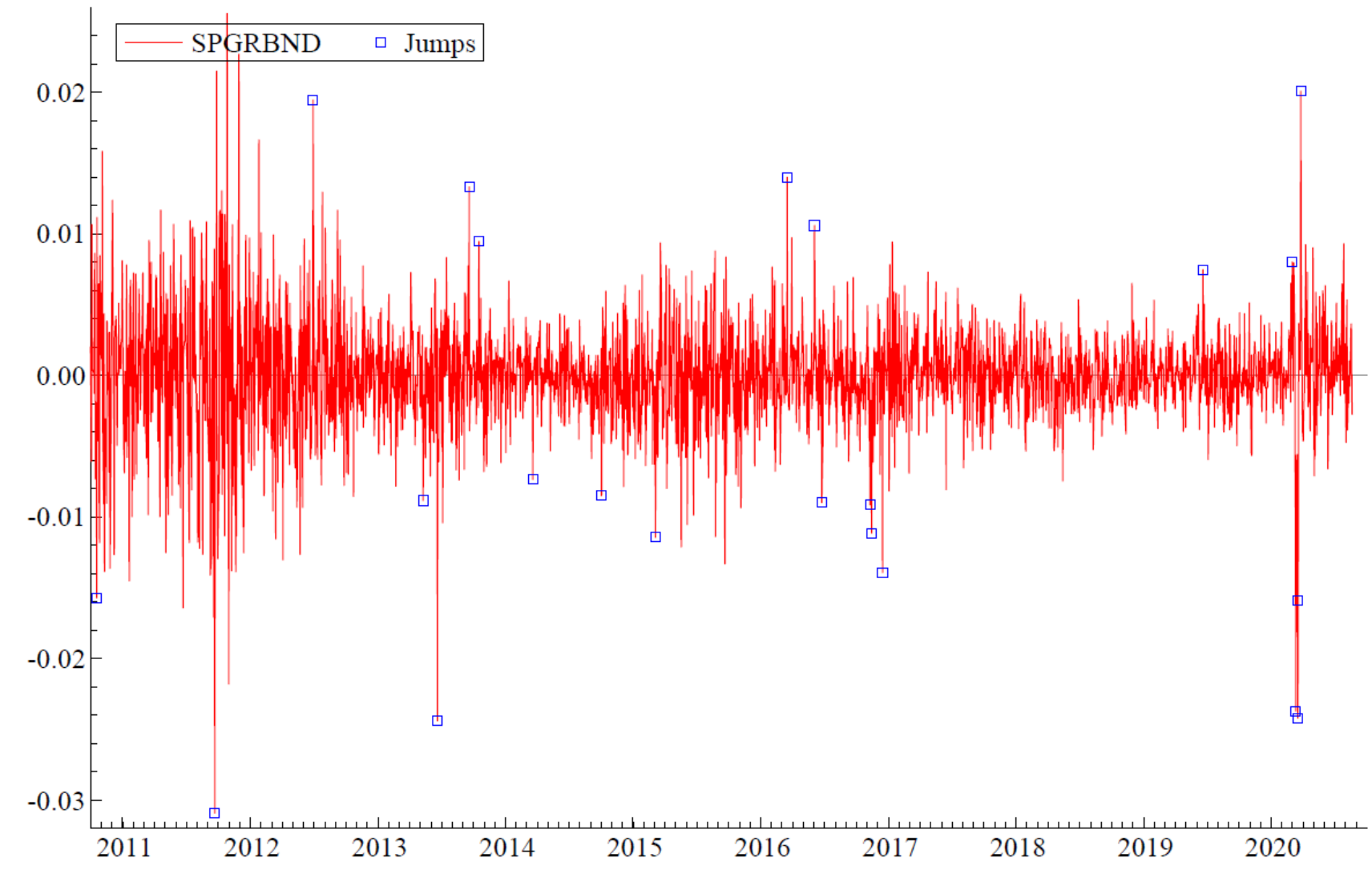


B) SPCOMP

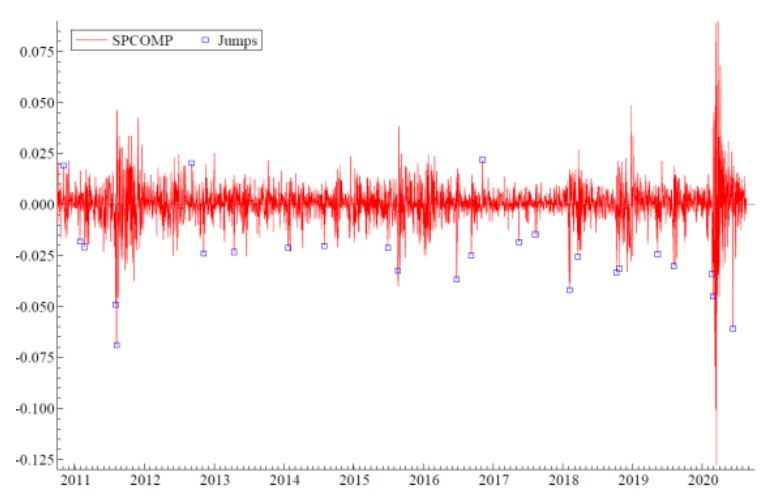

E) CHSASHR

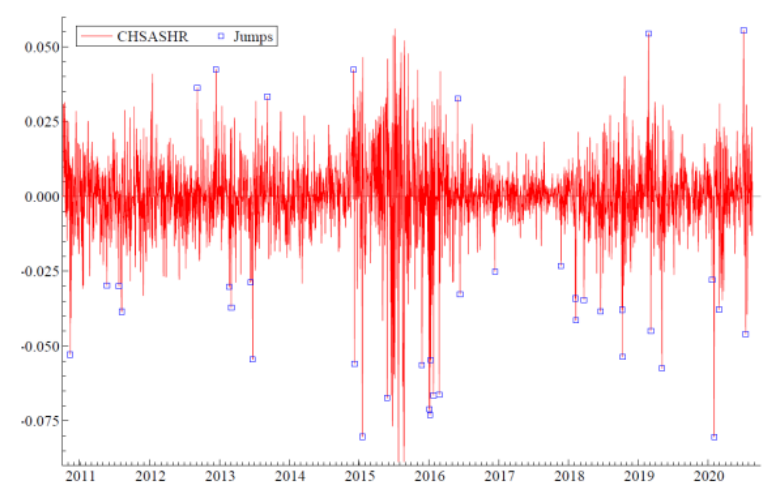

C) FTSE100

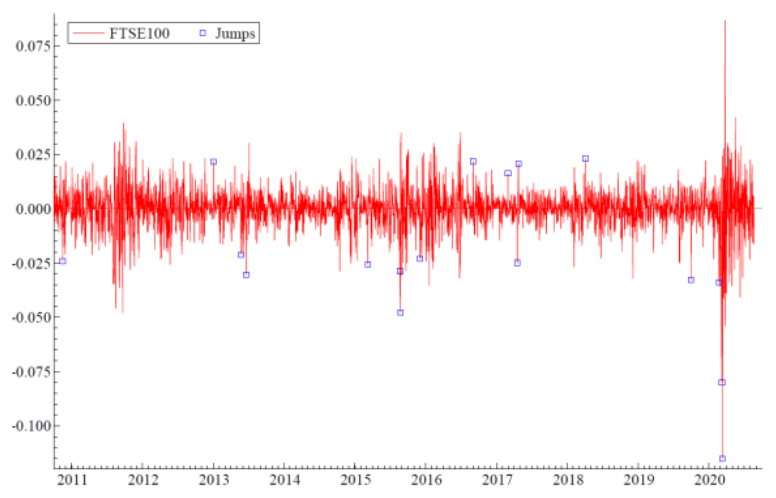

F) MSWRLD

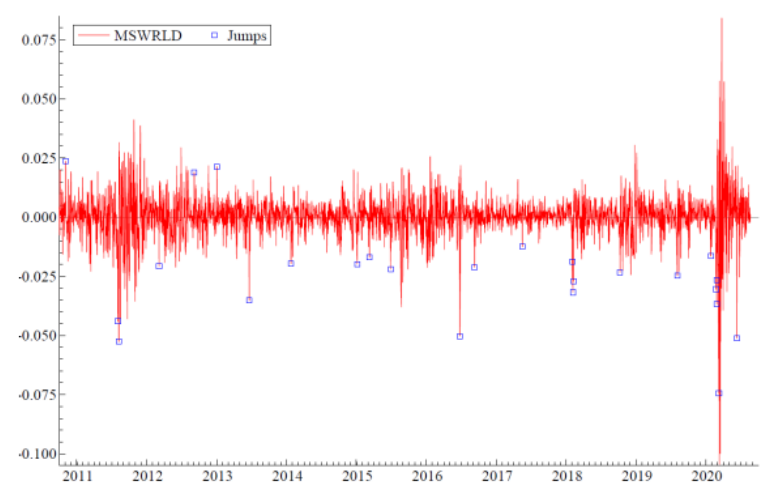

D) DAXINDX

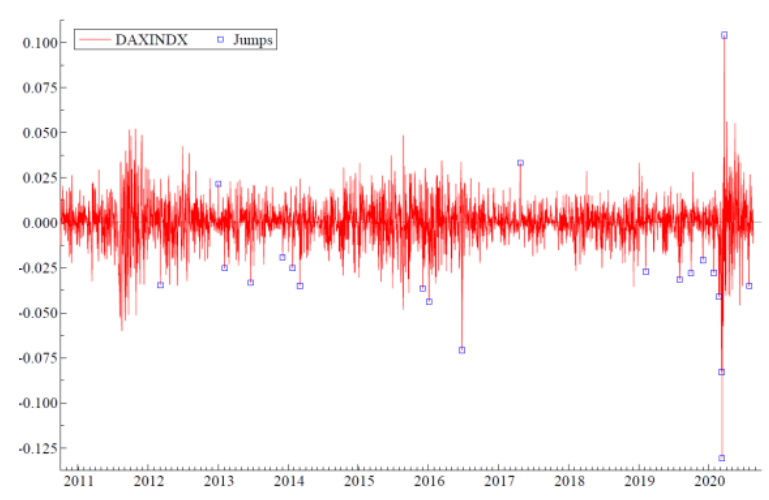

G) MSEROP

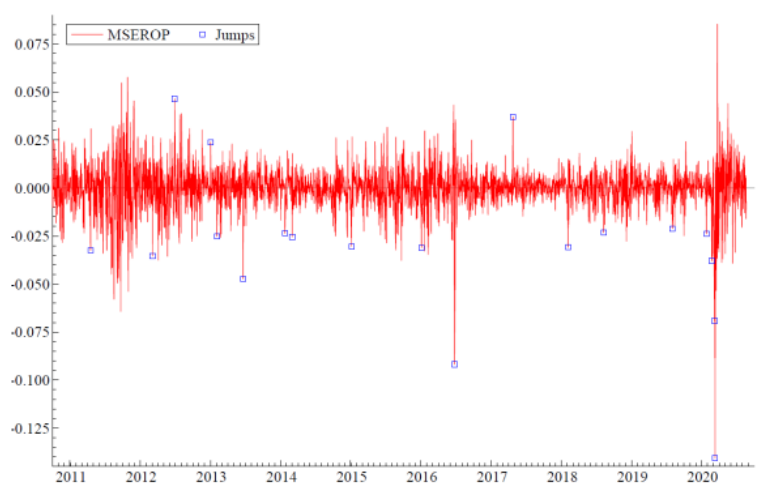

28 
H) MSPACF

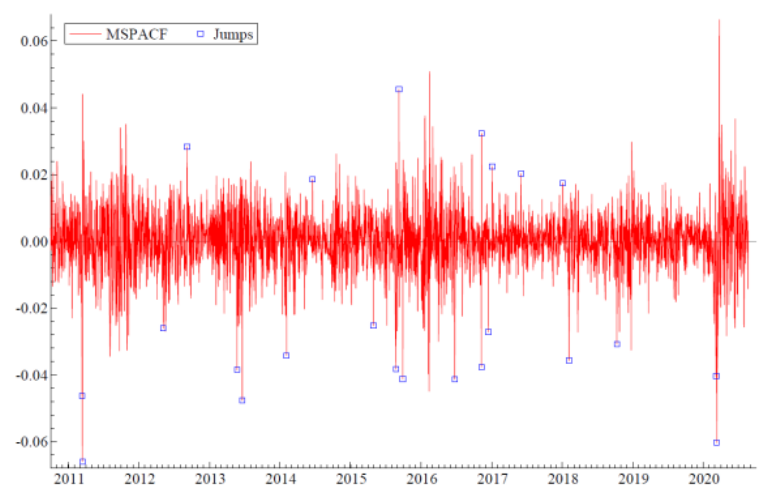

K) $\mathrm{OIL}$

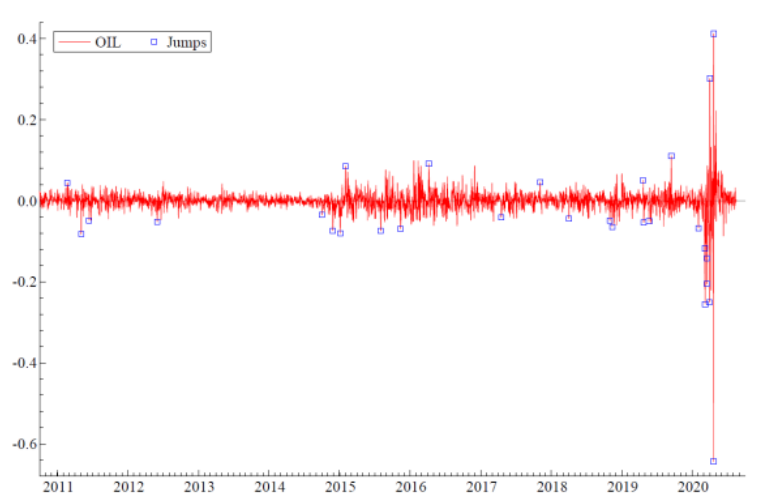

I) $\mathrm{BOND}$

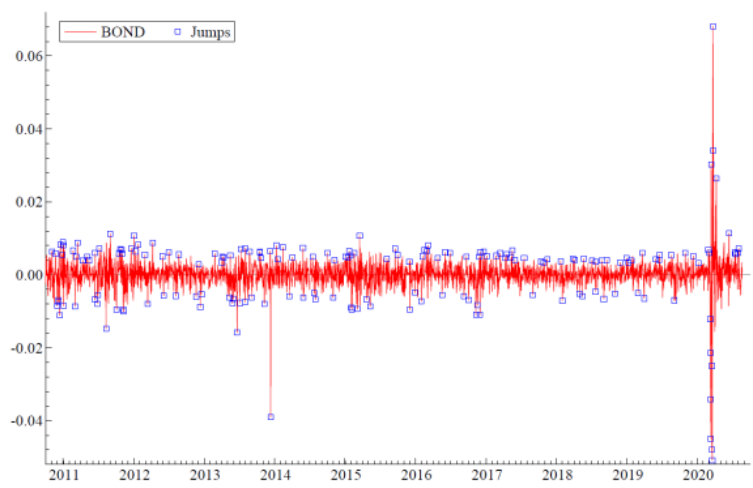

L) GOLD

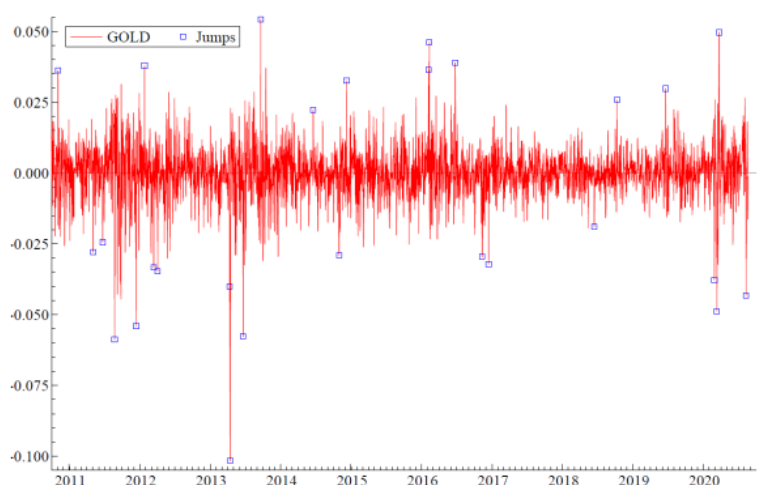

J) USDXY

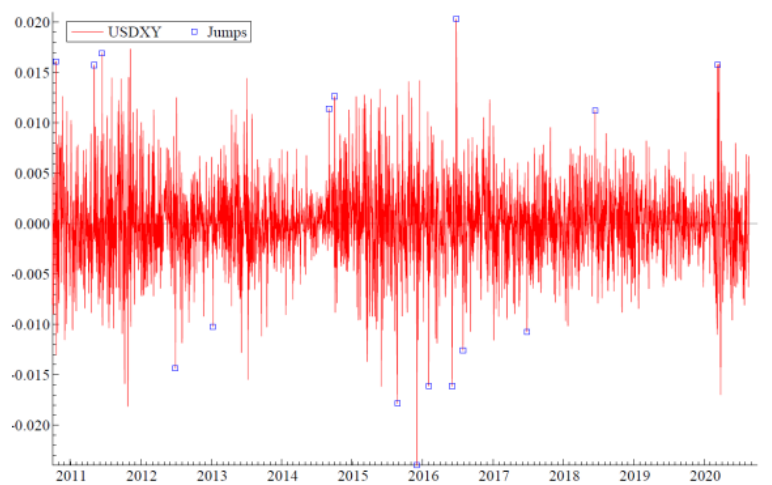

M) SPGSCI

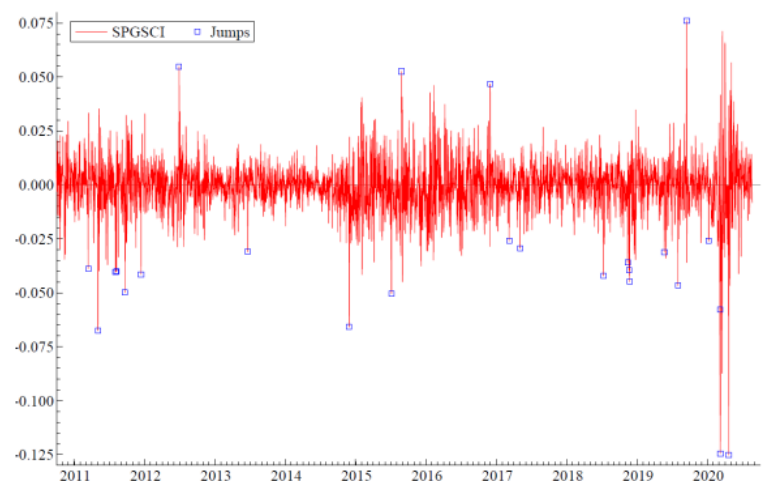

Fig. 1 These figures represent the plots of jumps on the return series of S\&P Green bonds and other financial markets. 


\section{Lag 1}

Lag 5

Lag 22

\section{A) SPCOMP}
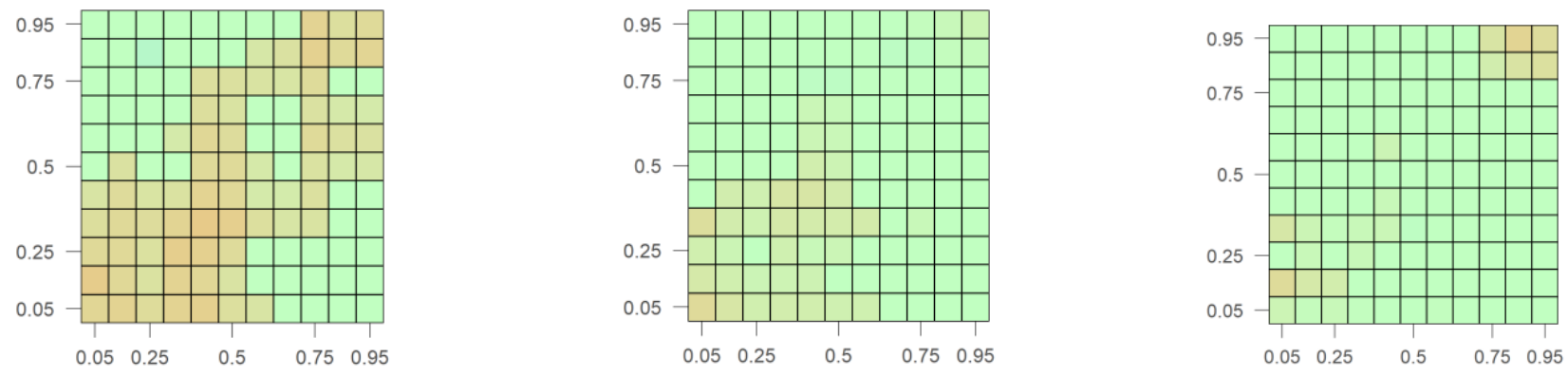

\section{B) FTSE100}
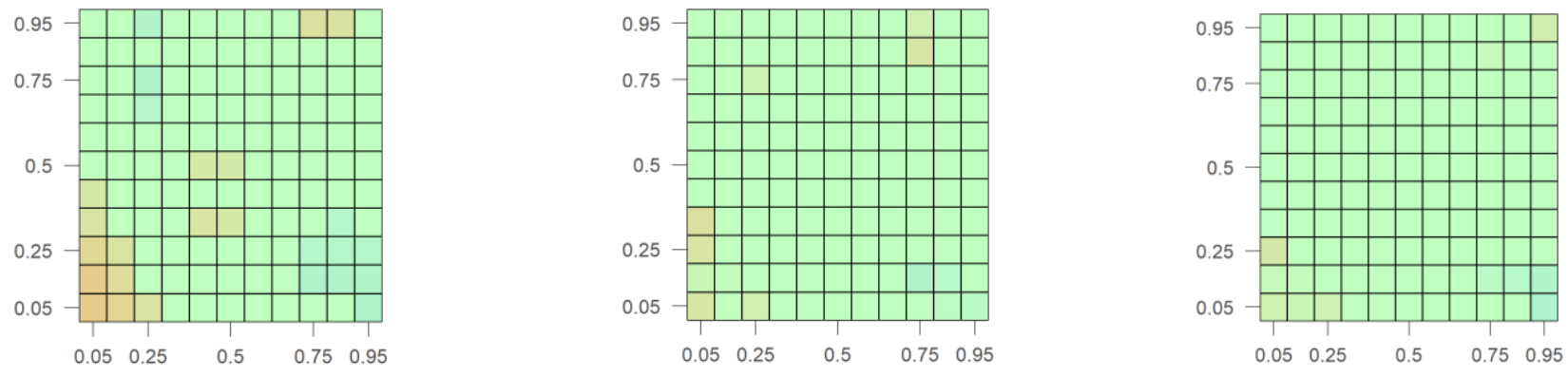

\section{C) DAXINDX}
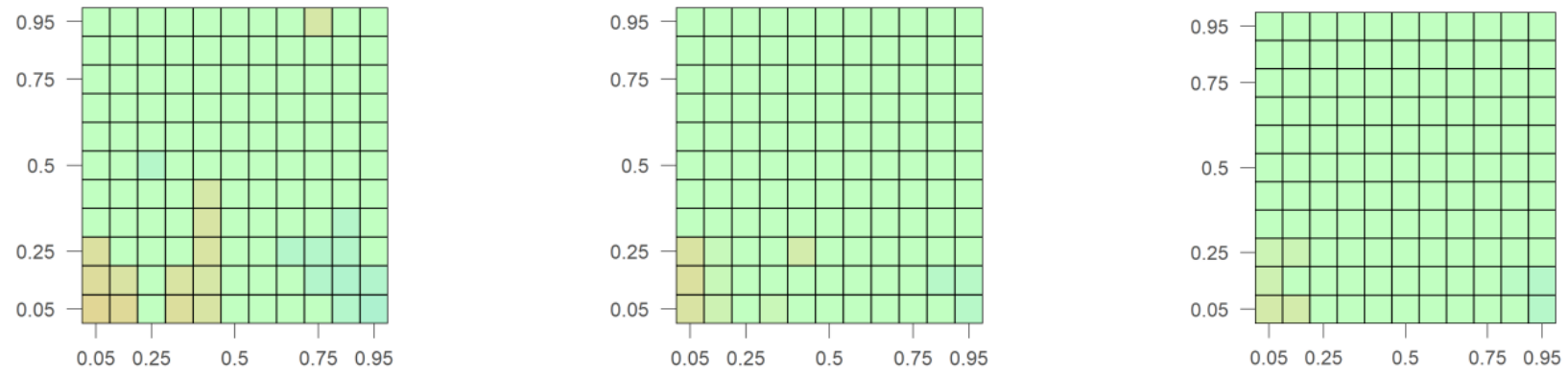

\section{D) CHSASHR}
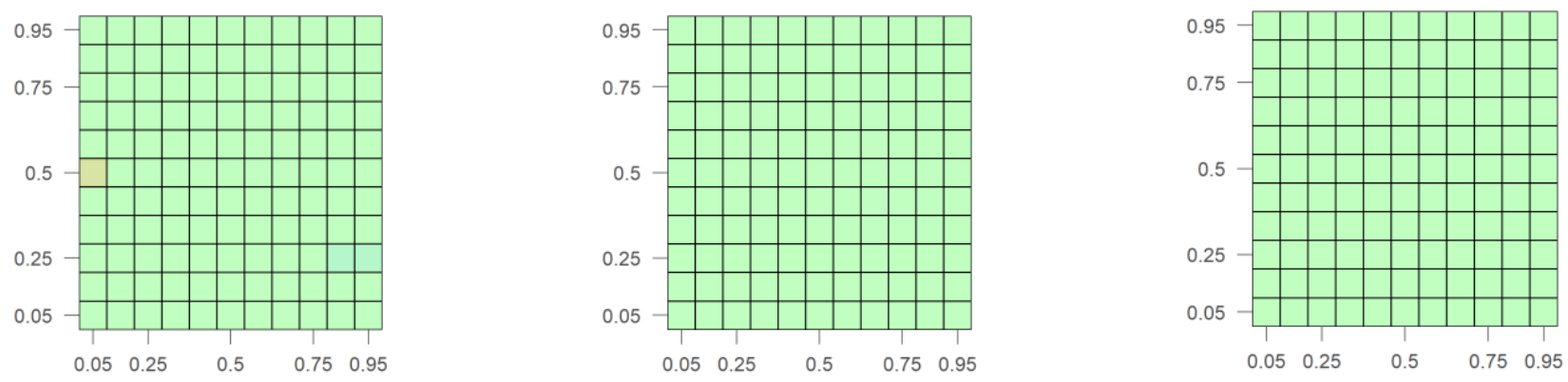


\section{E) MSWRLD}

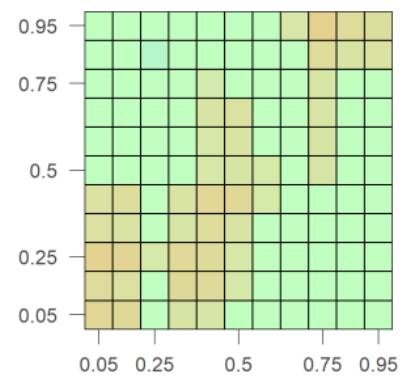

\section{F) MSEROP}

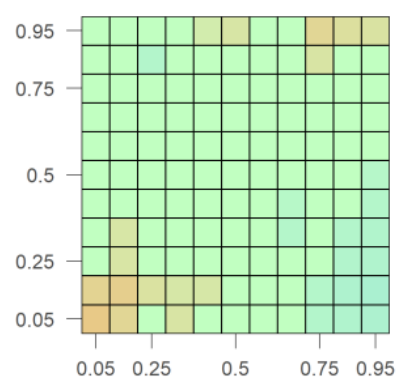

\section{G) MSPACF}

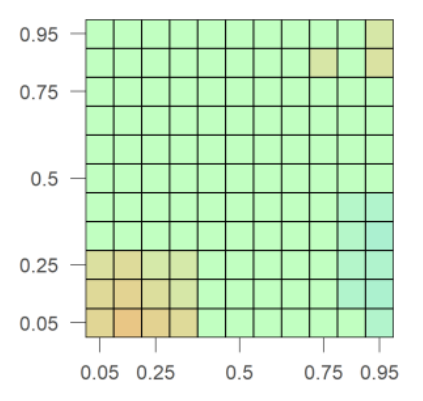

\section{H) BOND}

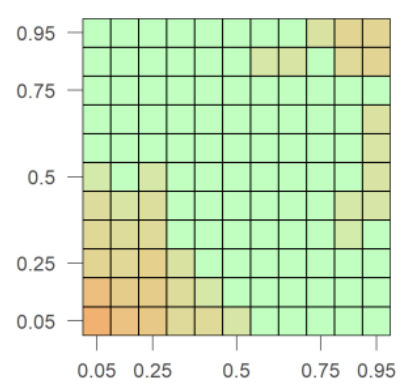

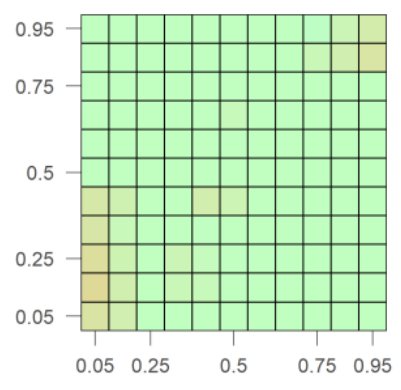
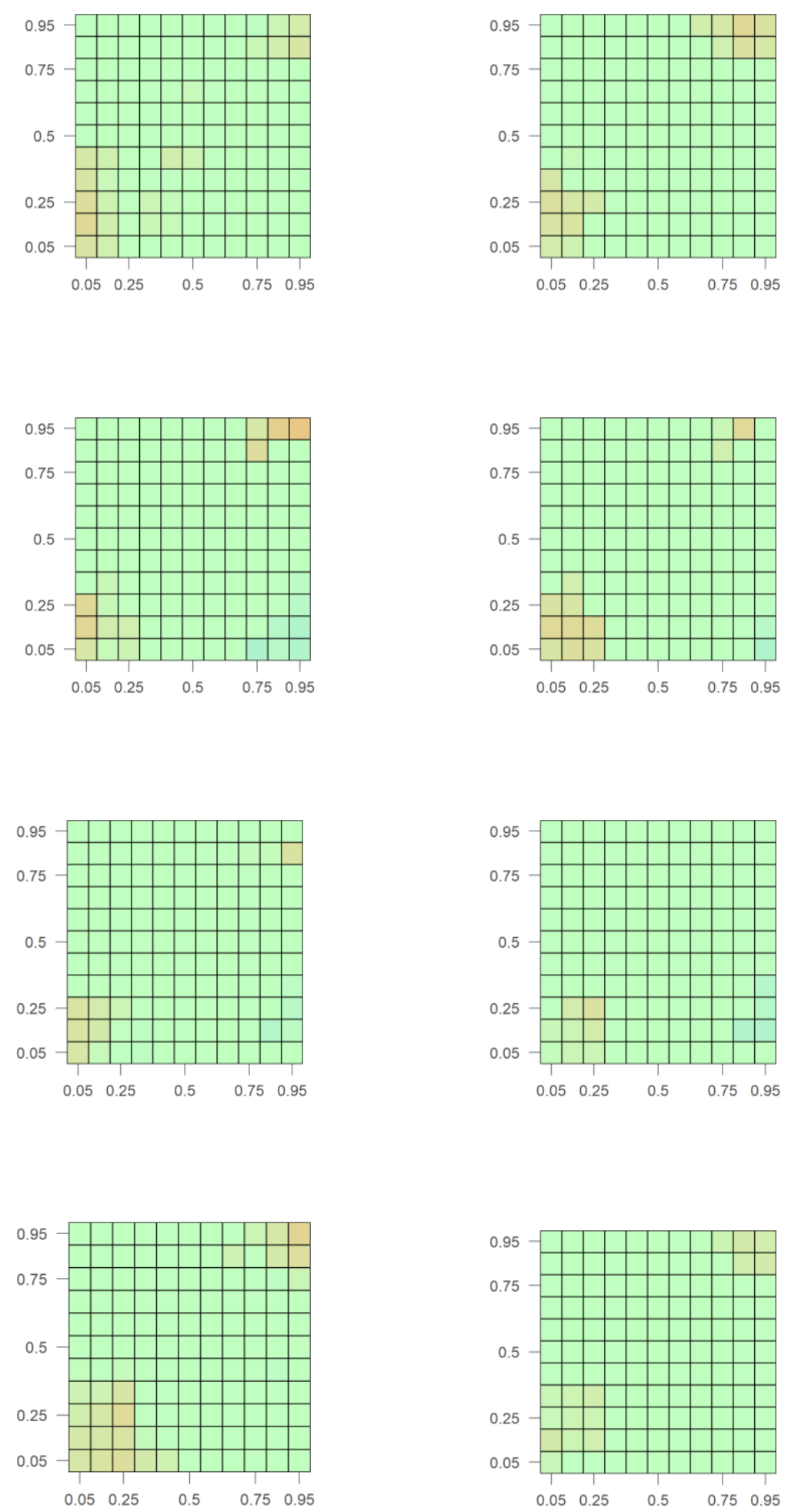

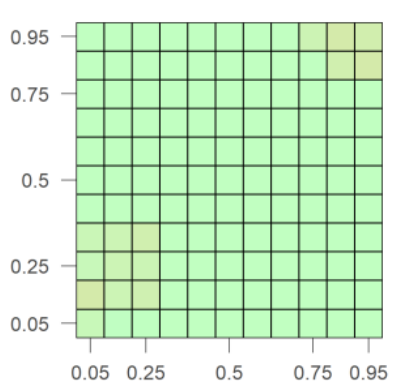




\section{I) USDXY}

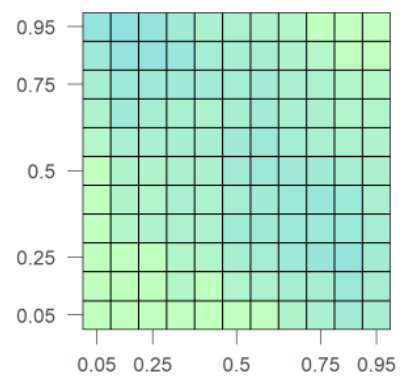

\section{J) SPGSCI}

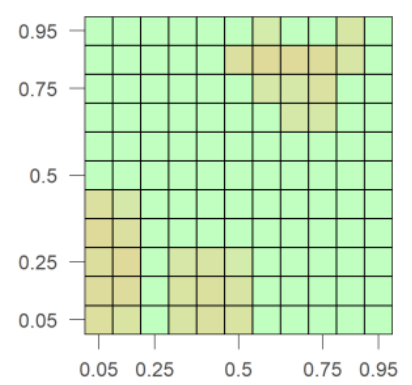

\section{K) OIL}

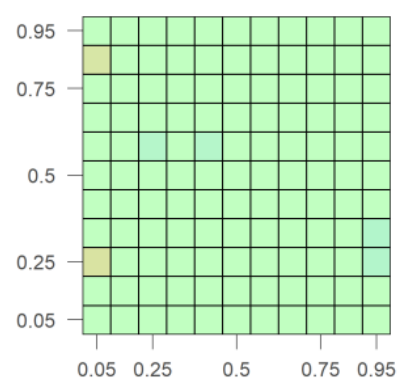

\section{L) GOLD}

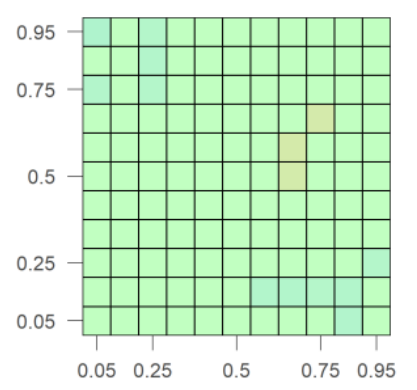

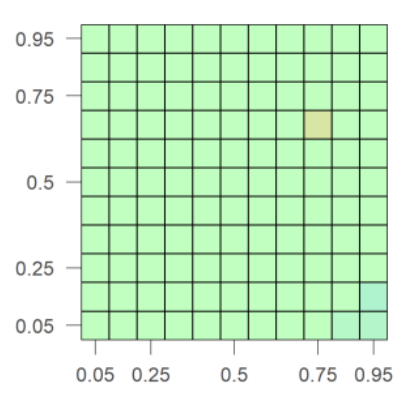
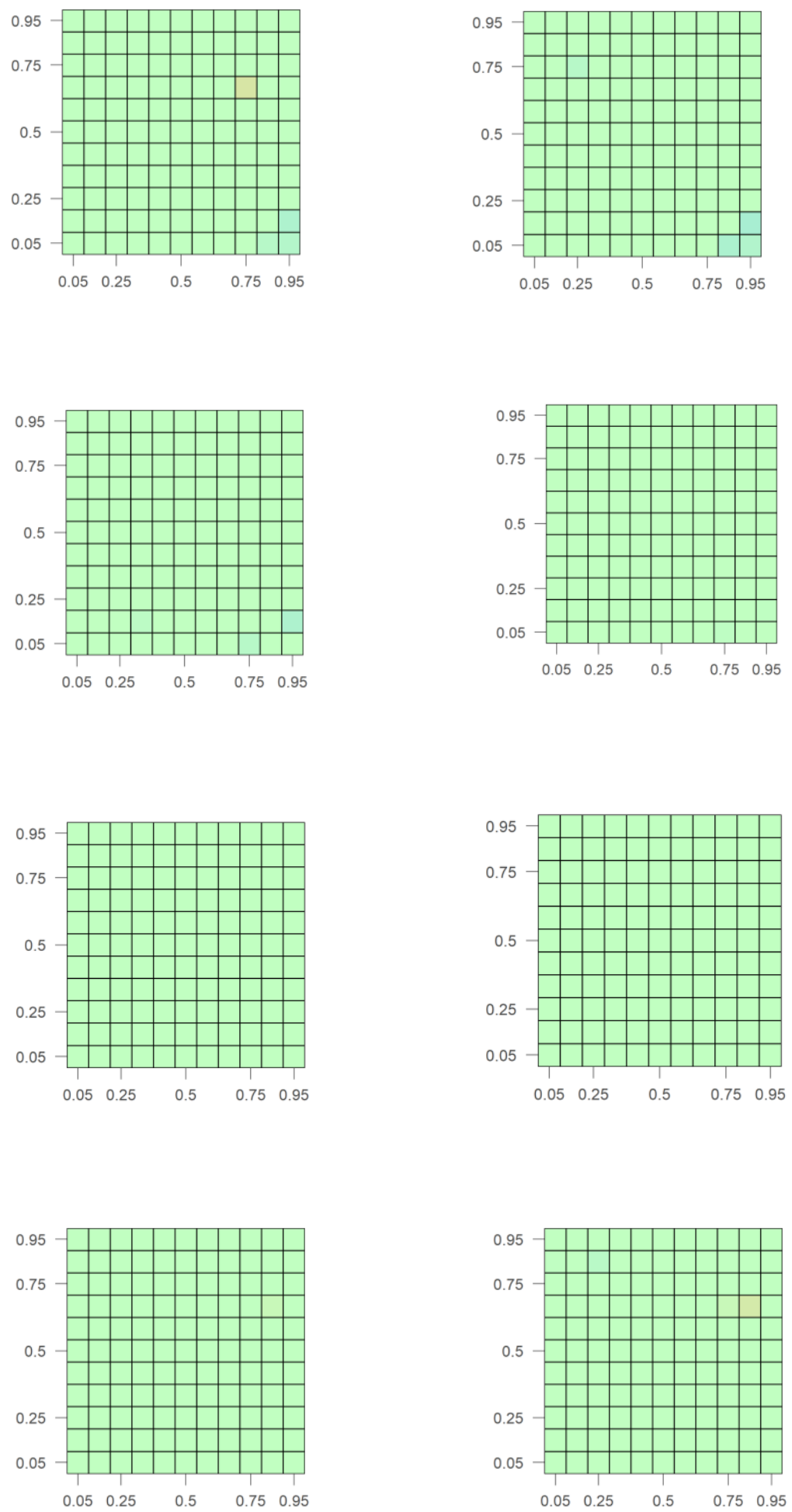

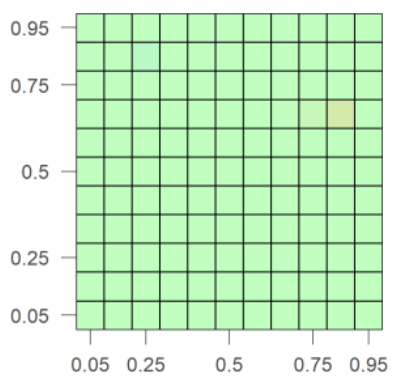

Fig. 2 Heat maps of CQ between daily S\&P Green Bond and Financial Markets - Full sample.

Note: These figures show the CQ in the form of heat maps. The quantile levels with no significant directional predictability are set to zero. The colored rectangles are the predictable regions where the Box-Ljung test statistic is statistically significant. In each heat map, the horizontal axis represents financial market return quantiles, while the vertical axis represents S\&P Green Bond return quantiles. 


\section{Lag 1}

\section{A) SPCOMP}
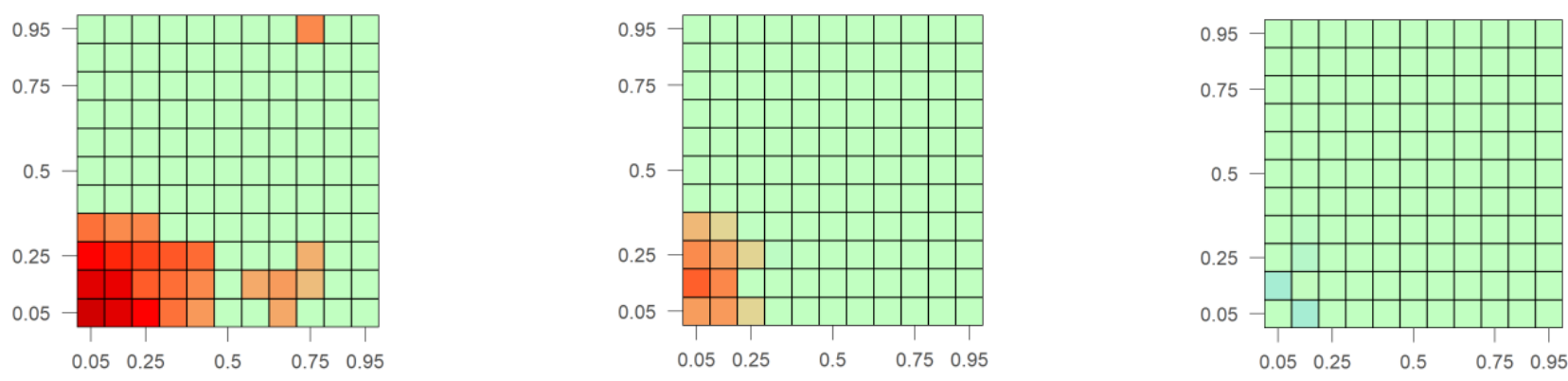

\section{B) FTSE100}
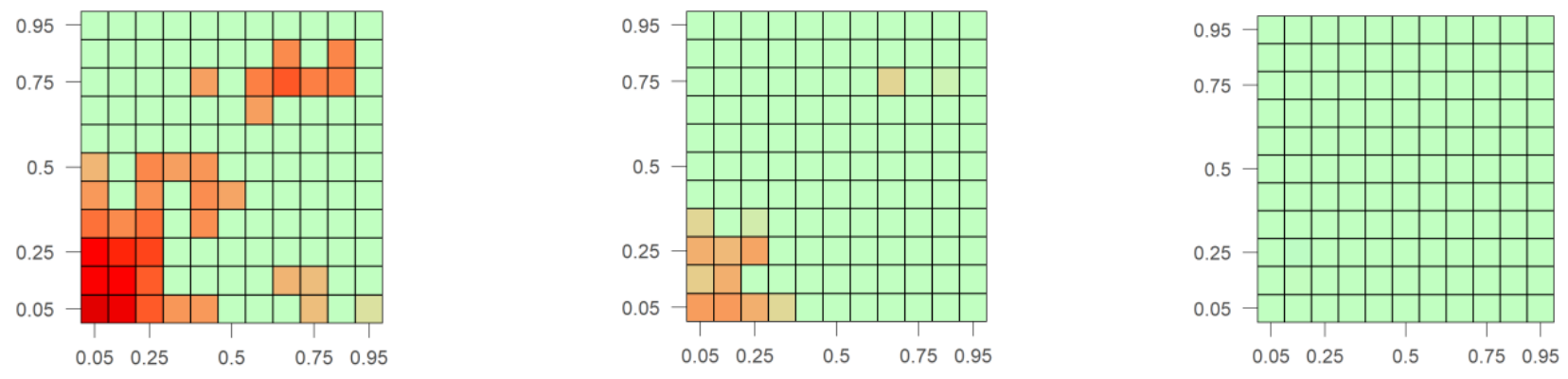

\section{C) DAXINDX}
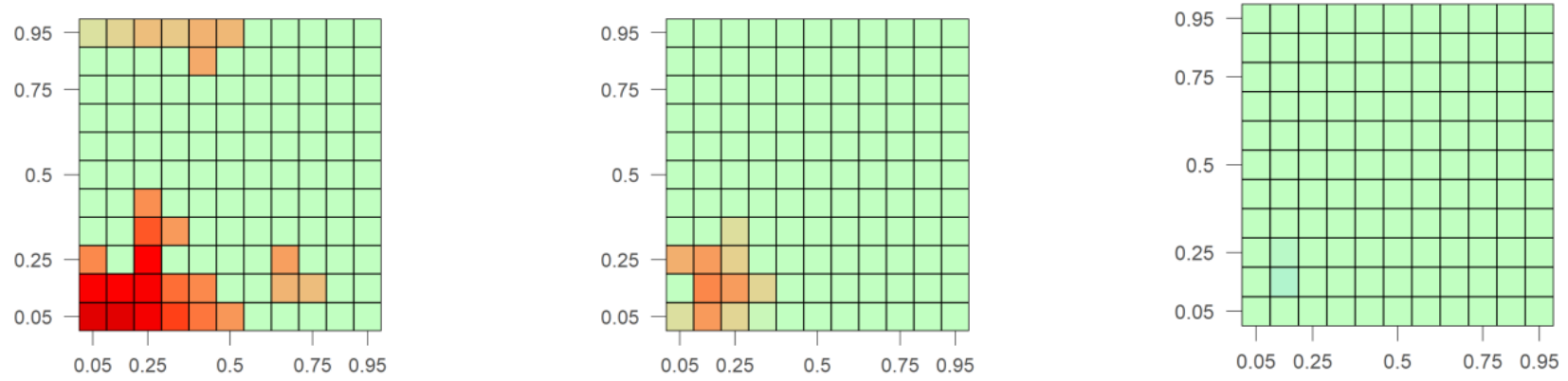

\section{D) CHSASHR}
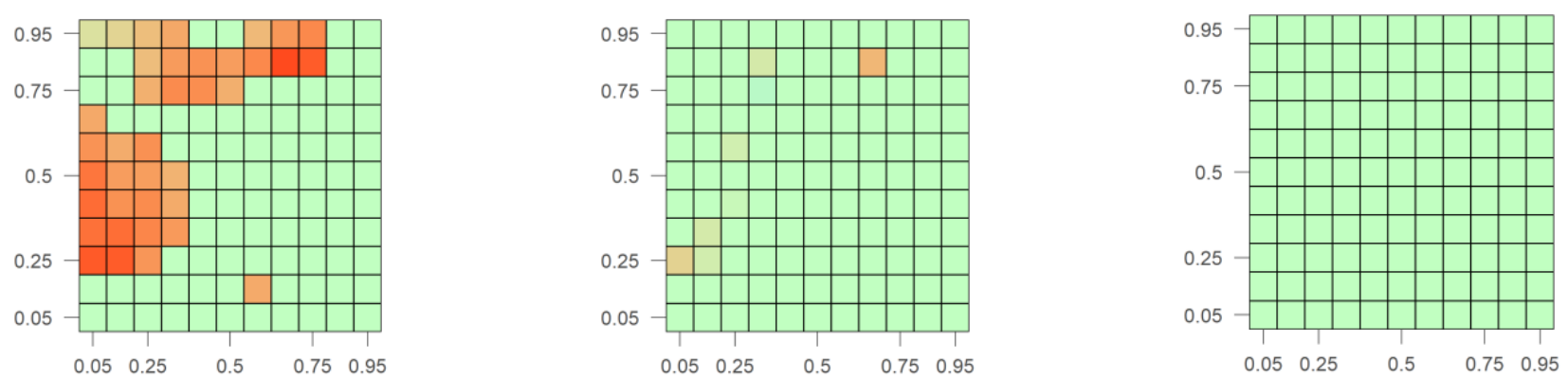
Lag 1

Lag 5

\section{E) MSWRLD}
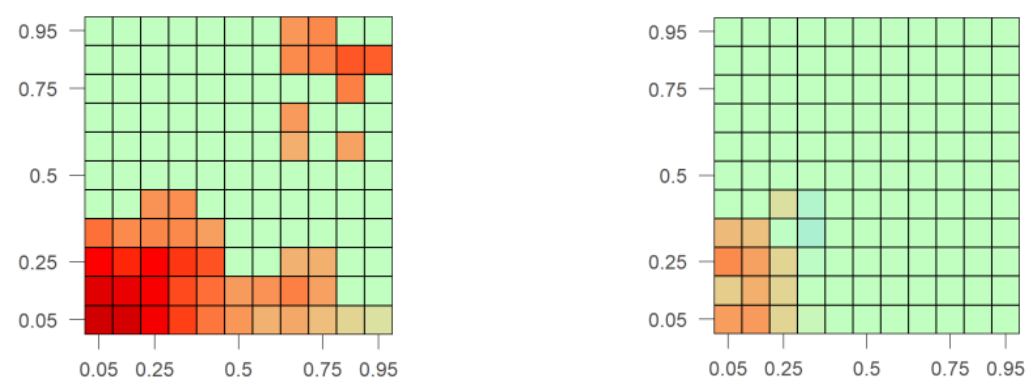

\section{F) MSEROP}
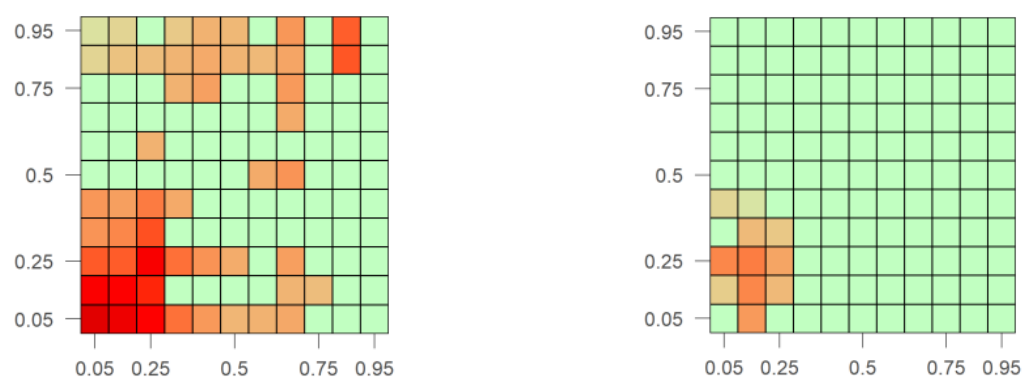

\section{G) MSPACF}

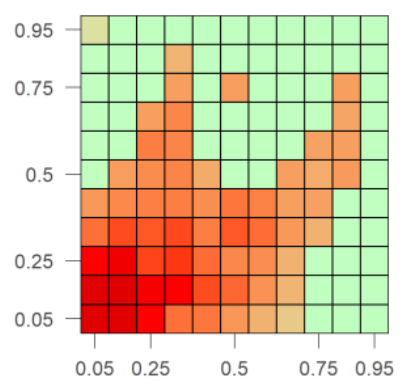

\section{H) BOND}

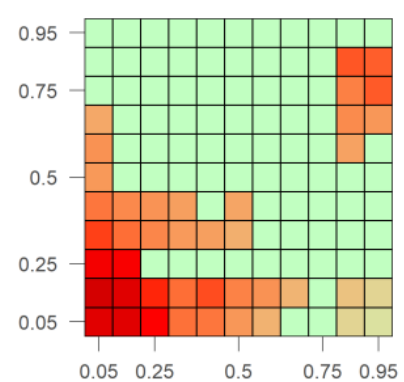

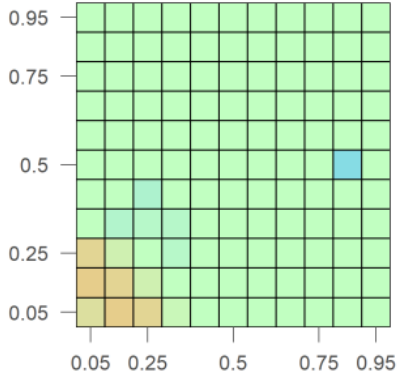

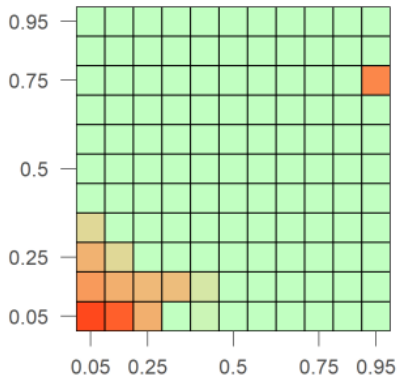

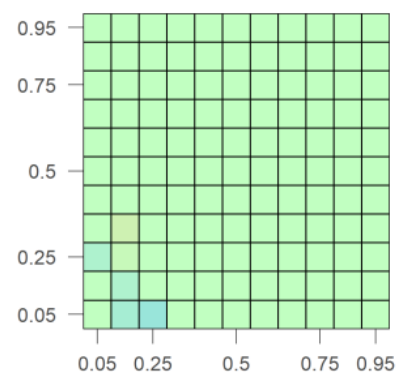

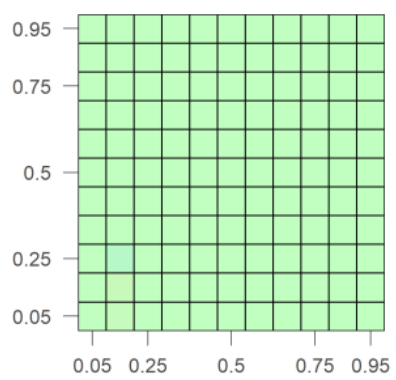

Lag 22

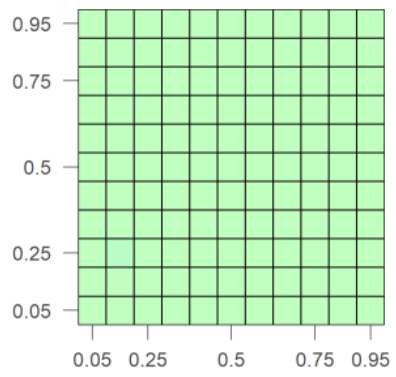

$\begin{array}{lllll}05 & 0.25 & 0.5 & 0.75 & 0.95\end{array}$

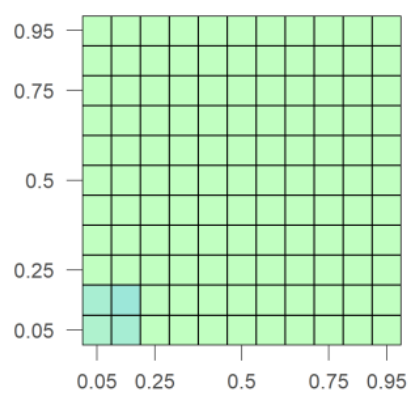




\section{I) USDXY}
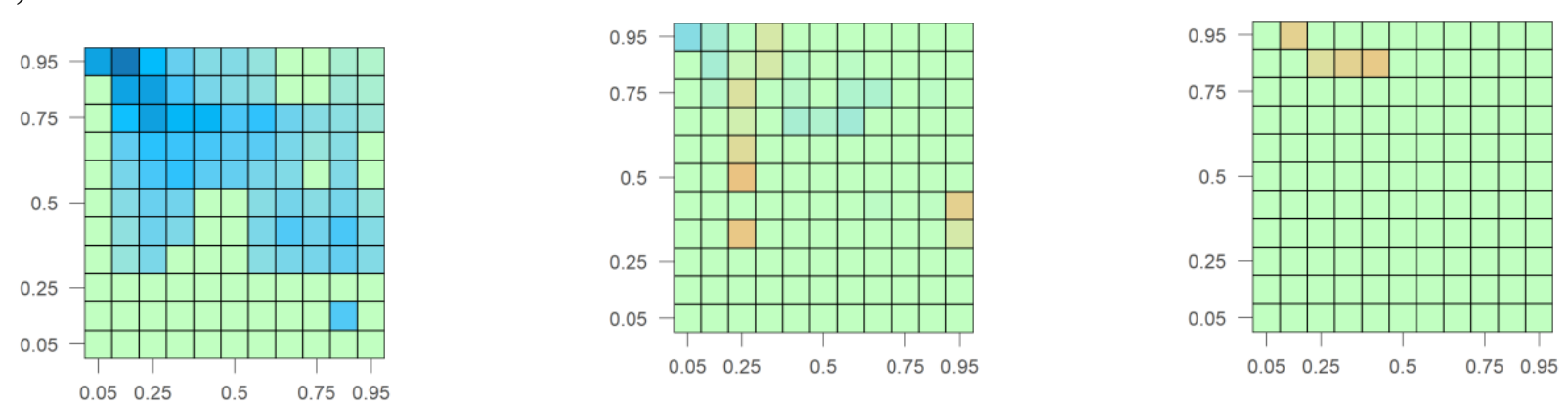

\section{J) SPGSCI}

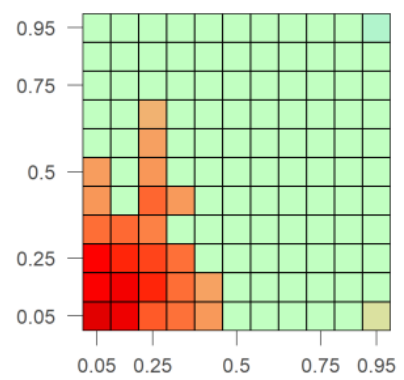

\section{K) OIL}

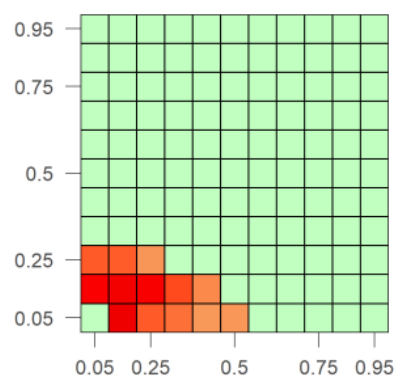

\section{L) GOLD}

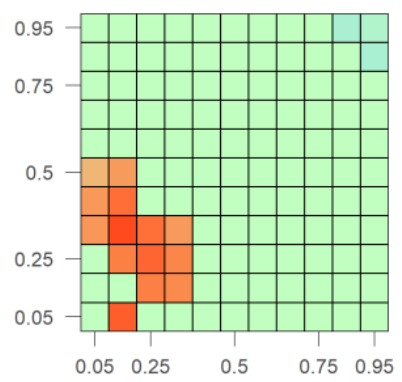

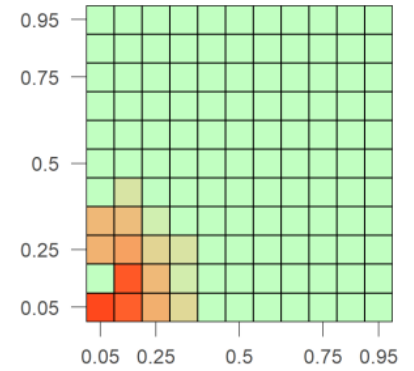
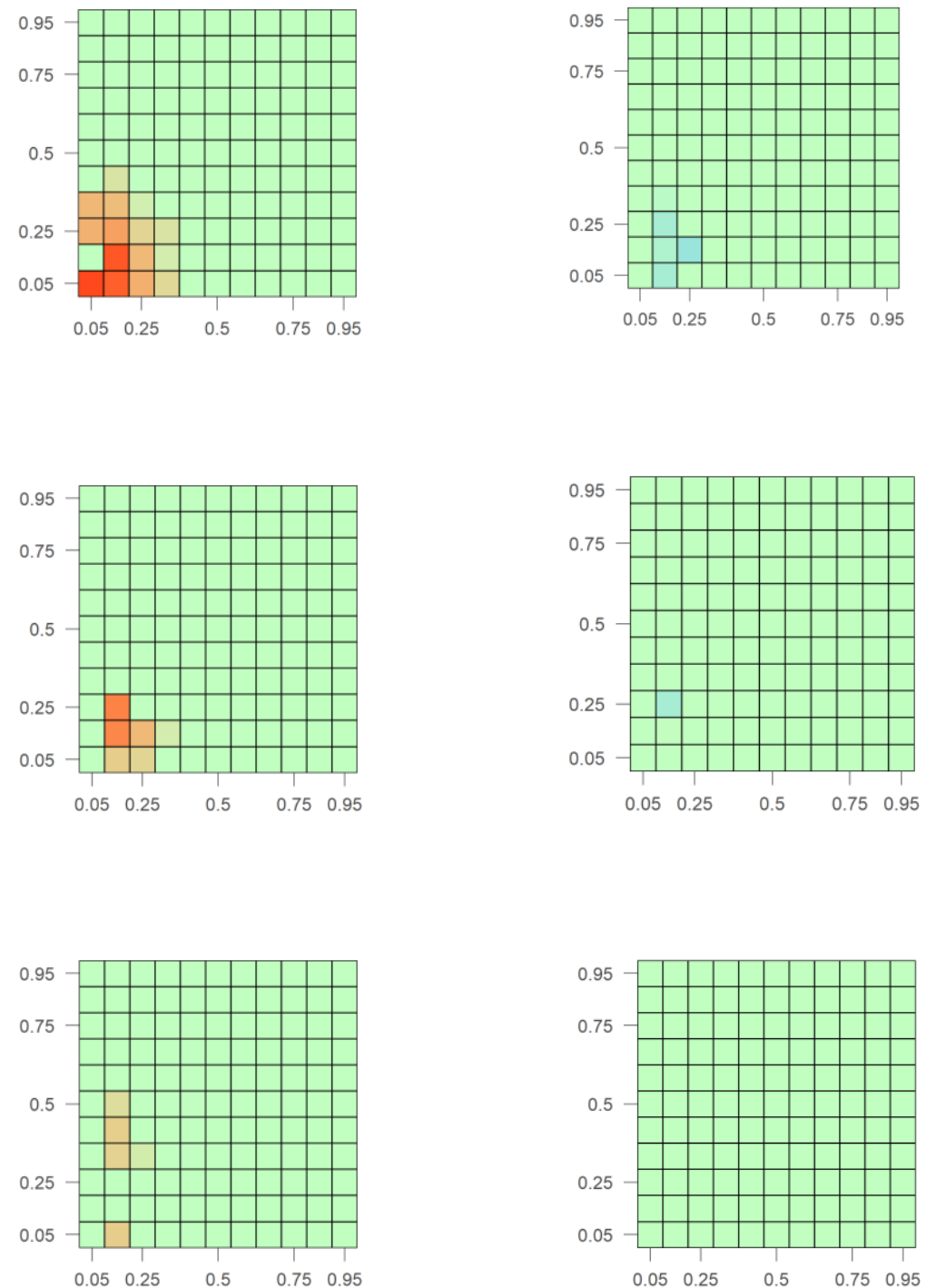

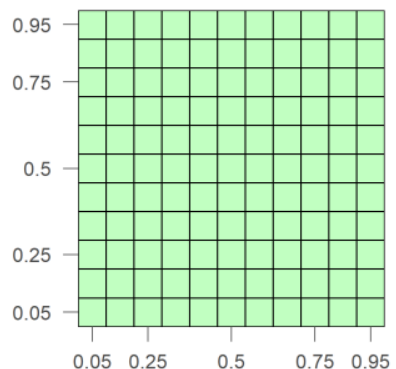

Fig. 3 Heat maps of CQ between daily S\&P Green Bond and Financial Markets - COVID sub-sample.

Note: These figures show the CQ in the form of heat maps. The quantile levels with no significant directional predictability are set to zero. The colored rectangles are the predictable regions where the Box-Ljung test statistic is statistically significant. In each heat map, the horizontal axis represents Financial market return quantiles, while the vertical axis represents S\&P Green Bond return quantiles. 


\section{A) SPCOMP}
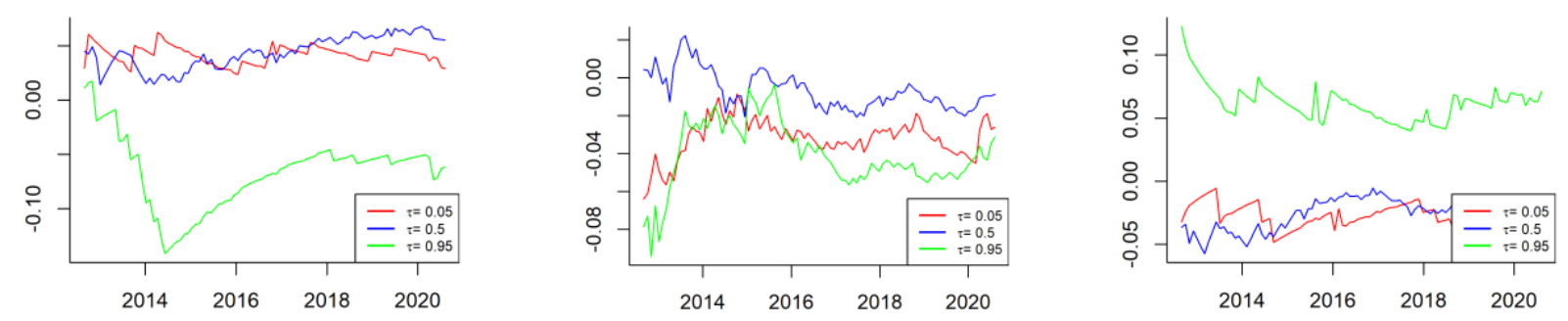

\section{B) FTSE100}
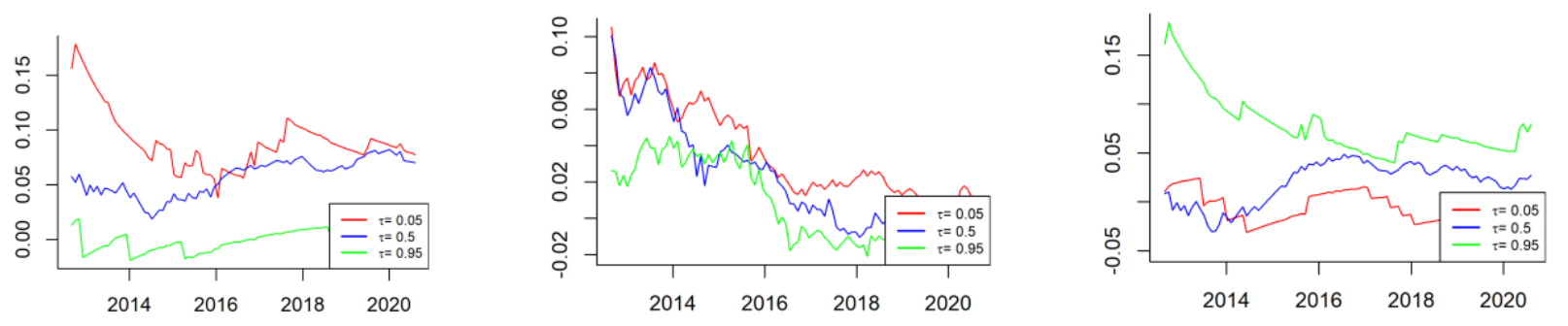

\section{C) DAXINDX}
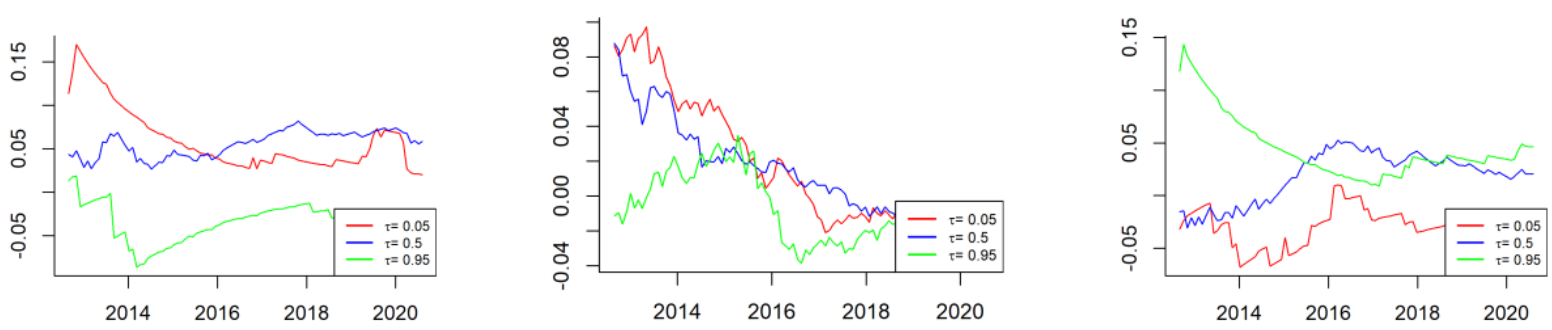

\section{D) CHSASHR}
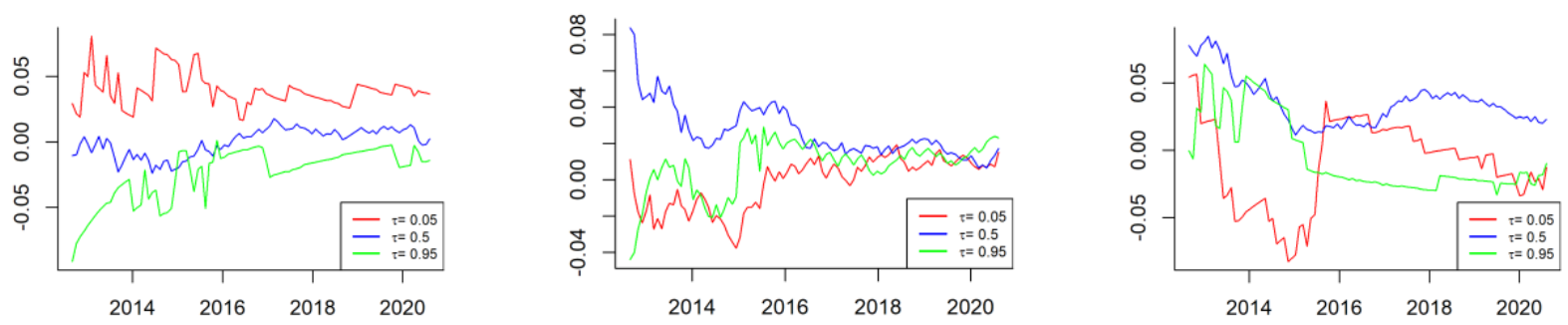


\section{E) MSWRLD}
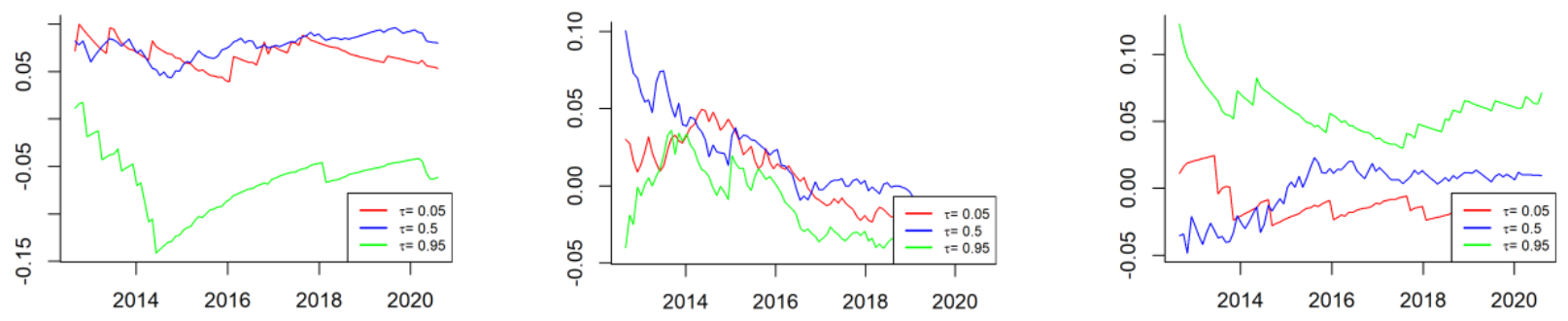

\section{F) MSEROP}
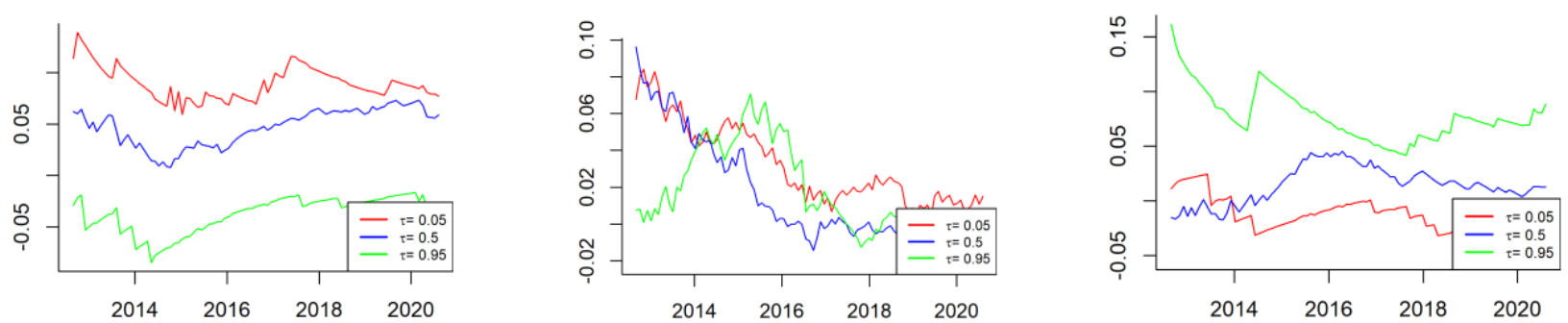

\section{F) MSPACF}
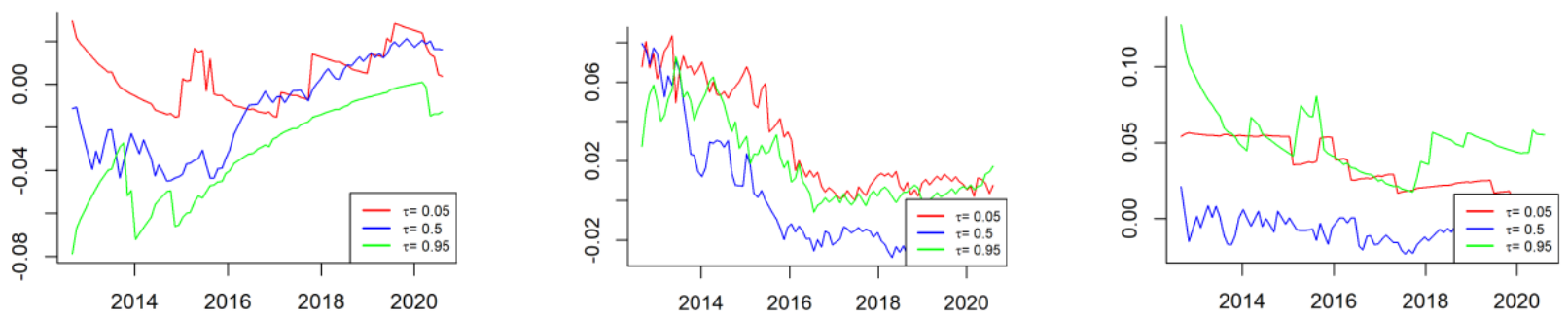

\section{F) BOND}
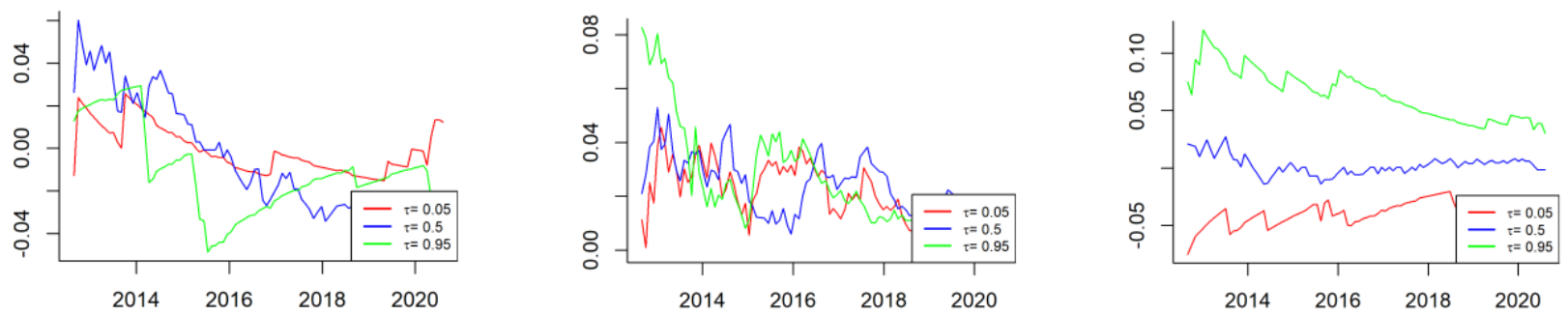


\section{I) USDXY}
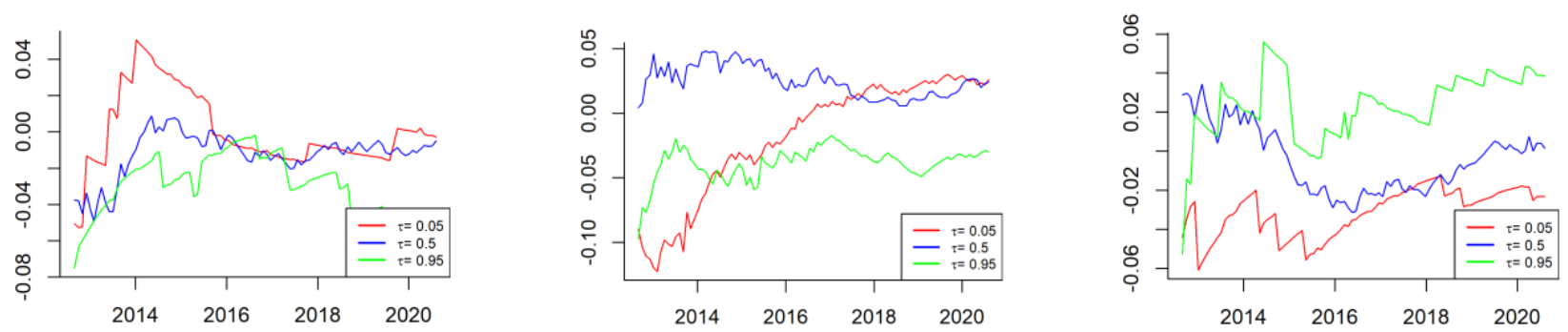

\section{J) SPGSCI}
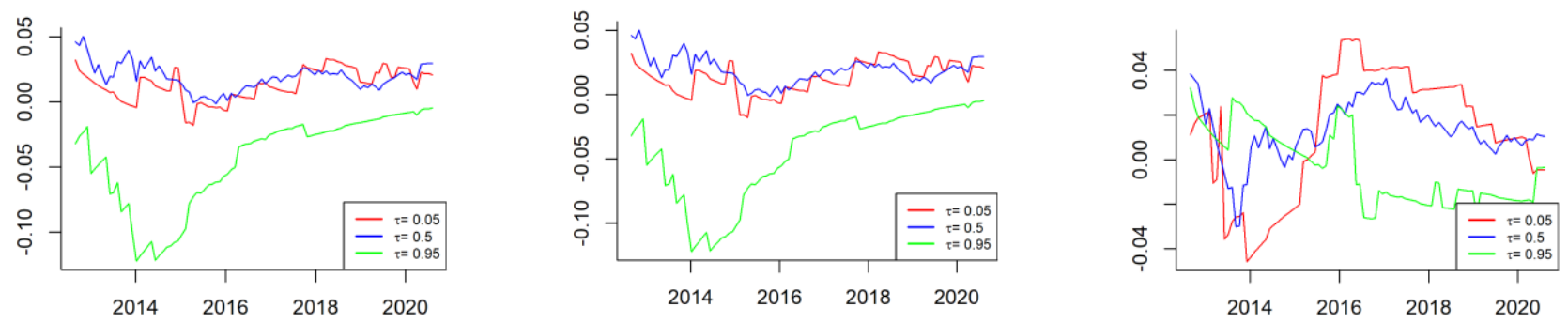

\section{K) OIL}
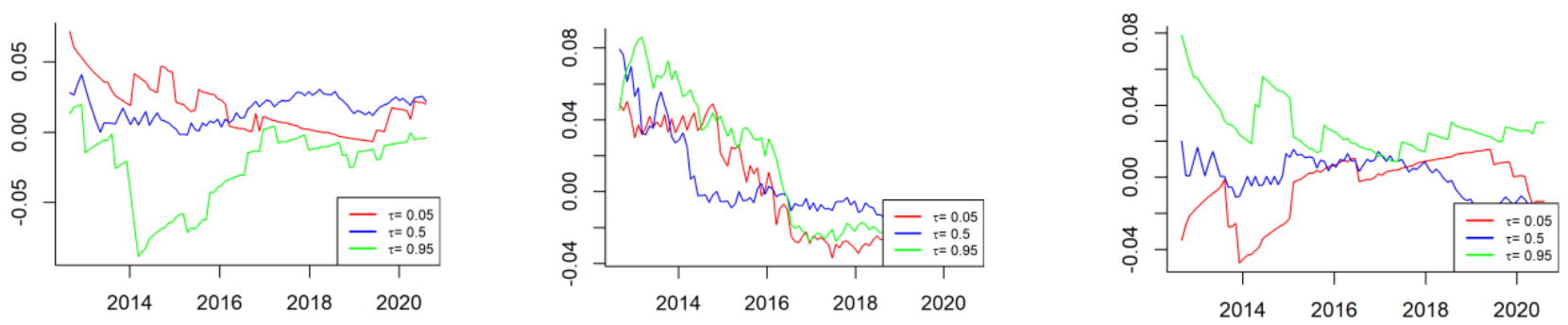

\section{L) GOLD}
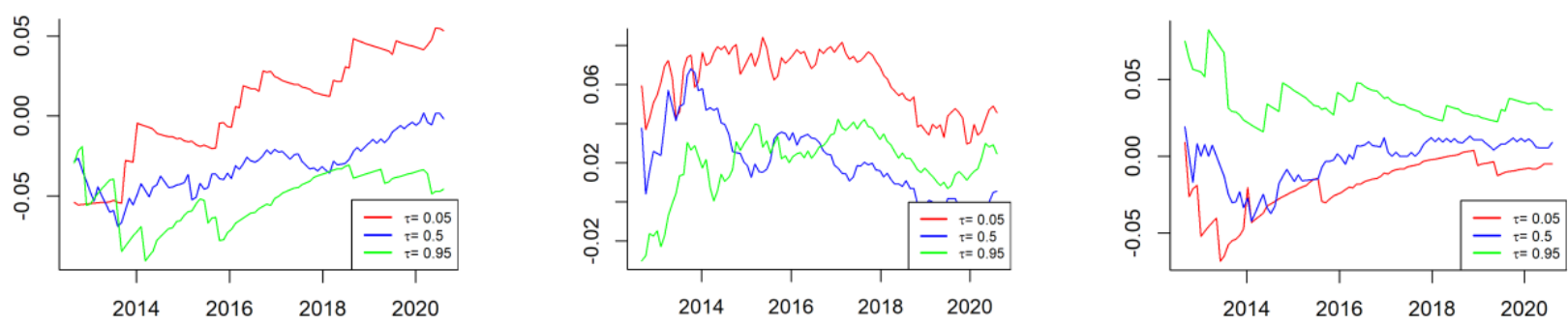

Fig. 4 Recursive CQ between daily S\&P Green Bond and Financial market returns.

Note: The vertical (horizontal) axis represents the quantile hits for the financial markets (time). The starting year of the rolling window is marked on the horizontal axis. The left, middle, and right columns, respectively, show the 5\%,50\%, and 95\% quantiles for S\&P Green Bond while, the red, blue, and green lines represent the 5\%,50\%, and 95\% quantiles for the financial market returns. Lag $\mathrm{p}=1$ 